Annals of Mathematics, 151 (2000), 459-515

\title{
Double bubbles minimize
}

\author{
By Joel Hass and Roger SchlaflY*
}

\begin{abstract}
The classical isoperimetric inequality in $\mathbb{R}^{3}$ states that the surface of smallest area enclosing a given volume is a sphere. We show that the least area surface enclosing two equal volumes is a double bubble, a surface made of two pieces of round spheres separated by a flat disk, meeting along a single circle at an angle of $120^{\circ}$.
\end{abstract}

\section{Introduction}

In this paper we find the unique surface of smallest area enclosing two equal volumes. The surface is called a double bubble, and is made of two pieces of round spheres separated by a disk, meeting along a single circle at an angle of $120^{\circ}$. This is the form assumed by two equally sized soap bubbles which are brought together until their boundaries conglomerate to form a common wall. See Figure 1, due to J. Sullivan.

Isoperimetric problems, which study maximizing the size of an enclosed bounded region whose boundary is of fixed size, are among the oldest problems in mathematics. For a broad discussion of isoperimetric problems see Osserman [33].

The two volume isoperimetric problem in $\mathbb{R}^{3}$ was considered by the Belgian physicist J. Plateau [35], and appears in C.V. Boys' famous book on soap bubbles. As Boys wrote,

"When however the bubble is not single, say two have been blown in real contact with one another, again the bubbles must together take such a form that the total surface of the two spherical segments and of the part common to both, which I shall call the interface, is the smallest possible surface which will contain the two volumes of air and keep them separate."

\footnotetext{
*The first author was partially supported by the National Science Foundation.
} 


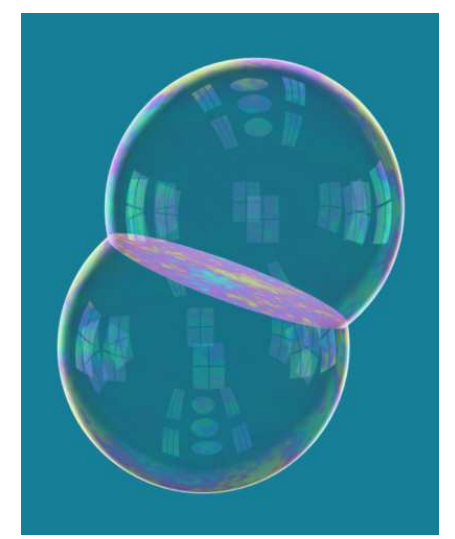

Figure 1. A double bubble enclosing two equal volumes.

We now outline briefly the recent history of the problem, with more details given later. F. Almgren proved the existence of bubble clusters enclosing a given collection of volumes in three-space and minimizing area among a general class of surfaces. J. Taylor established a regularity result for these bubble clusters, showing that they satisfy regularity properties physically observed by Plateau. B. White suggested an argument for showing that a two-region minimizer must be a surface of revolution, and J. Foisy and M. Hutchings filled in the details of this argument. At this point it was known that the minimizer consisted of pieces of Delaunay surfaces meeting at $120^{\circ}$. Hutchings went on to eliminate many remaining configurations, leaving just the standard double bubble and an additional family known as torus bubbles as the potential minimizers. In this paper we closely examine the family of torus bubbles and show they cannot be minimizers for the equal volume double bubble problem. Each torus bubble is determined by an ODE, and we can rigorously eliminate the entire collection by an examination of the solutions of these ODEs using a computational technique called interval arithmetic, described in Section 5. At the end, only the standard double bubble remains as a possible minimizer.

Extensive interest in the problem was generated in recent years by work of F. Morgan. The planar case has been solved in [1] by methods special to two dimensions. A fundamental paper of Almgren [3] established the existence of solutions to a great variety of geometric minimization problems, including multiple component isoperimetric problems in $\mathbb{R}^{3}$. Despite the fact that existence was established some time ago, our result is the first explicit example of a surface in $\mathbb{R}^{3}$ solving a multiple region finite volume isoperimetric problem. Lawlor and Morgan have shown that the cone over the regular tetrahedron is a minimizing surface among all those with its boundary that separate the four faces of the tetrahedron, but it is still not known whether this surface is minimizing without the separation condition. 
Such multiple region isoperimetric problems arise in many fields, including the growth and shape of biological cells [28], [40]. There were extensive studies of such problems by physical and biological scientists in the $19^{\text {th }}$ century [24], [35]. Plateau established experimentally that a soap bubble cluster is a piecewise-smooth surface having only two types of singularities. The first type of singularity occurs when three smooth surfaces come together along a smooth triple curve at an angle of $120^{\circ}$. The second type of singularity occurs when six smooth surfaces and four triple curves converge at a point. The angles are then equal to those of the cone over the 1-skeleton of a regular tetrahedron. A mathematical proof that these types of singularities are the only ones possible in a minimizing bubble in $\mathbb{R}^{3}$ was given by Taylor [39]. However there were no explicit minimizing bubbles known for any collection of volumes exhibiting either of these singularities. Thus we have found the first explicit example of a closed minimizing surface in $\mathbb{R}^{3}$ known to exhibit some of the singularities predicted by Plateau.

The arguments presented in this paper are a mixture of geometrical analysis and of estimates of geometric quantities obtained by the use of numerical computation. We perform these calculations with strict estimates on the accuracy of the computations. Since it is still somewhat unusual in a mathematical proof to use digital computers to do calculations involving real numbers, we will say a few words about the nature of this part of the argument.

Computers are widely known in the mathematical community to have been used successfully for analysis of discrete and combinatorial problems, such as the 4-color theorem [6], [37]. Problems such as isoperimetric inequalities are qualitatively different, since they inherently involve real numbers. Real numbers are represented in digital computers in a floating point format which allows exact description of only a finite number of rationals. Simple calculations, like division, or even addition, lead to unrepresentable numbers, and so the computer must round off. For purposes of mathematical proof, the size of the round-off must be accurately tracked throughout the calculation.

Methods for strictly estimating solutions of differential equations exist, but are not yet widely used in the mathematical community. The vast majority of numerical work is for approximation and simulation that does not meet the standards of a mathematical proof. However there have been some important results achieved through rigorous use of floating point numerical methods to achieve traditional mathematical proofs, notably Lanford's work on the Feigenbaum Conjectures [27] and Fefferman and de la Llave's work on the stability of matter [12], [15] and the work of McKay and Percival on nonexistence of invariant tori [34]. See also recent work of Hales on the Kepler Conjecture [19] and Gabai, Meyerhoff and Thurston [17] on hyperbolic 3 -manifolds. Computer calculations are essential to our proof that equal vol- 
ume double bubbles minimize area. There are too many calculations to be done by hand.

The proof parameterizes the space of possible solutions by a two-dimensional rectangle, one dimension corresponding to an angle and the other to a mean curvature. This rectangle is subdivided into 15,016 smaller rectangles which are investigated by calculations involving a total of 51,256 numerical integrals. Every calculation is done with strict error bounds, and all results are precise mathematical statements. All operations conform to the IEEE 754 standard for computer arithmetic, a widely adopted standardized method for implementing real number (floating point) computations on computers [5]. Our methods indicate that numerical techniques are likely to play an important role in future geometrical arguments.

The main result is the following, announced in [20]:

THEOREM 1. The unique surface of least area enclosing two equal volumes in $\mathbb{R}^{3}$ is a double bubble.

Corollary 2. For any surface in $\mathbb{R}^{3}$ enclosing two regions, each of volume $v$, the area a satisfies

$$
a^{3} \geq 243 \pi v^{2}
$$

with equality if and only if the surface is a double bubble enclosing two regions of volume $v$.

The first issue in establishing Theorem 1 is to find an appropriate category of surface in which to minimize area.

It suffices in this paper to consider piecewise smooth two-dimensional surfaces. It is sometimes useful to consider a much more general notion of surface, such as the $(F, \varepsilon, \delta)$ sets described in [3]. Our arguments actually imply that the double bubble minimizes in this larger class; see Theorem 2.2. Consideration of such a larger class of surfaces is needed primarily in the establishment of the existence and regularity of a minimizer, carried out in [3] and [39], and we will not need to be overly concerned with it in this paper.

Define a piecewise-smooth curve to be an embedded finite union of smooth curves, with any two either disjoint or having intersection contained in their endpoints. Define a piecewise-smooth surface to be an embedded finite union of smooth surfaces with piecewise-smooth boundary curves, with any two surfaces either disjoint or intersecting along piecewise-smooth curves contained in their boundaries. The set of points which are not in the interior of a smooth subsurface of a piecewise-smooth surface is called the singular set.

Define a bubble to be a piecewise-smooth surface satisfying:

1. Each two dimensional surface has constant mean curvature. 
2. The singular set is of the type described by Plateau. It consists of smooth triple curves along which three smooth surfaces come together at an angle of $120^{\circ}$ and isolated vertices where six smooth surfaces and four triple curves converge at a point. The angles at the point are equal to those of the cone over the 1-skeleton of a regular tetrahedron.

3. The mean curvatures around an edge in the singular set where three surfaces have common boundary sum up to zero.

The above conditions are necessary for no local perturbation of a piecewisesmooth surface to decrease the area while preserving the volume in each of its complementary regions.

A bubble enclosing regions of prescribed volumes is called a minimizing bubble if it minimizes area among all bubbles enclosing the same volumes. The regions are not necessarily connected.

Given positive constants $v_{1}$ and $v_{2}$, let $a\left(v_{1}, v_{2}\right)$ denote the infimum of the area of piecewise-smooth surfaces enclosing two regions $R_{1}$ and $R_{2}$ in $\mathbb{R}^{3}$ which are closed bounded sets with disjoint interiors such that volume $\left(R_{1}\right)=v_{1}$ and volume $\left(R_{2}\right)=v_{2}$. We will refer to the interior of the complement of $R_{1} \cup R_{2}$ as the exterior region $R_{0}$. Note that $R_{0}$ may not be connected, in which case the bubble encloses some compact "empty regions".

A double bubble enclosing volumes $v_{1}, v_{2}$ is a surface made of three pieces of round spheres, meeting along a single circle at an angle of $120^{\circ}$, and enclosing two connected regions having volumes $v_{1}$ and $v_{2}$. We consider the plane to be a sphere of infinite radius in this setting, allowing the interface of a double bubble to be a flat disk, as occurs when $v_{1}$ and $v_{2}$ are equal. See Figure 2 . In this and all other figures, we assume that the axis of rotational symmetry is the $x$-axis. Furthermore, we will always take the generating curves for a surface of revolution to be in the upper half of the $x y$-plane.

It has been conjectured since the work of Plateau that double bubbles give the most efficient shape for enclosing two given volumes.

Conjecture 3. The double bubble enclosing volumes $v_{1}, v_{2}$ is the unique surface having area equal to $a\left(v_{1}, v_{2}\right)$.

Theorem 1 solves this conjecture in the case that $v_{1}=v_{2}$.

Deep results of Almgren and Taylor, summarized in Section 2, imply that for any two positive numbers $v_{1}, v_{2}$ there exists a minimizing bubble $S\left(v_{1}, v_{2}\right)$ in $\mathbb{R}^{3}$ which encloses volumes $v_{1}$ and $v_{2}$. Arguments based on ideas of $\mathrm{B}$. White and F. Morgan, and developed in [16], [22], [32], show that any solution must be a surface of revolution. We will need to refer to this proof, so we present a simple version for the case of two regions in $\mathbb{R}^{3}$ in Theorem 2.6. The lack of such an argument for isoperimetric problems involving three or more regions in $\mathbb{R}^{3}$ makes those problems more formidable. 
While the reduction to a surface of revolution gives an enormous simplification in the scale of the problem, F. Morgan has pointed out several major topological obstacles to solving the double bubble conjecture. The first is that the regions $R_{1}$ and $R_{2}$ bounded by the minimizing bubble $S\left(v_{1}, v_{2}\right)$ may not be connected, as illustrated in Figure 3.

A second problem is that $S\left(v_{1}, v_{2}\right)$ may enclose bounded regions in $\mathbb{R}^{3}$ which do not form part of either $R_{1}$ or $R_{2}$, as in Figure 4 . These regions are called empty regions by Morgan.
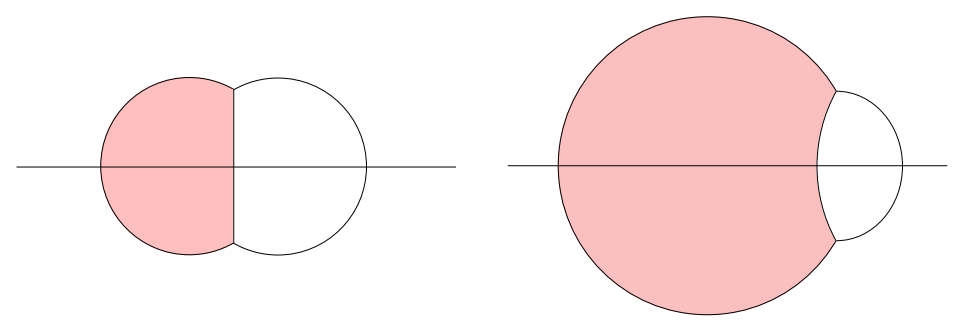

Figure 2. Equal and unequal volume double bubbles in cross-section. The axis of revolution is the $x$-axis.

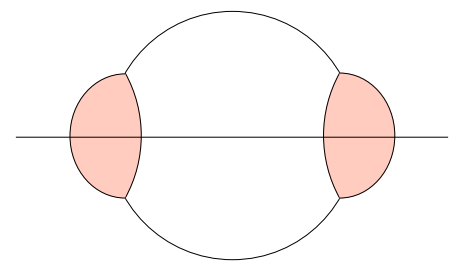

Figure 3. Cross-section of a bubble with a nonconnected region. The nonconnected region is shaded.

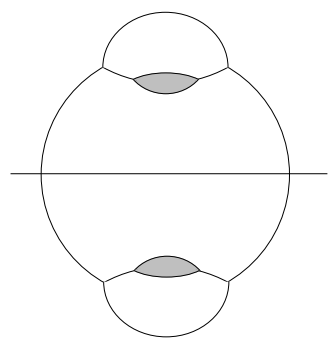

Figure 4. Cross-section of a bubble with an empty torus region. The shaded region generates a solid torus when revolved around the $x$-axis. 
A third problem is that $S\left(v_{1}, v_{2}\right)$ may enclose nonsimply connected regions. Since the surface is a surface of revolution, these regions are homeomorphic to solid tori, and we call them torus components.

There are therefore numerous possible configurations which a minimizing bubble might take. A recent breakthrough due to Hutchings [22] has given restrictions on the type of surfaces that can arise in the double bubble problem, eliminating many possibilities. Some remaining possibilities are depicted in Figure 5. For the case of equal volumes, Hutchings showed that there were further constraints. Each region must be connected, leaving only two possible configurations, the double bubble and an additional class of possibilities, called torus bubbles, whose properties will be discussed in Section 4 .
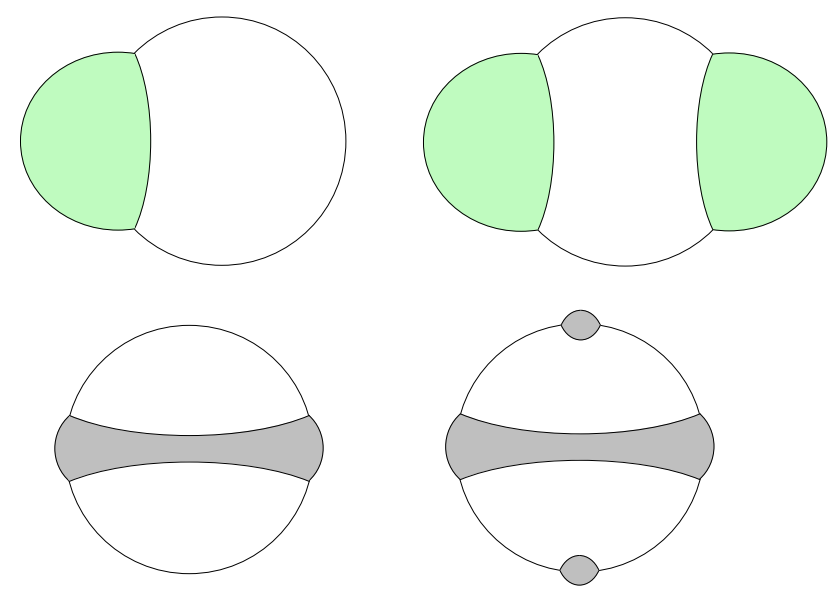

Figure 5. Cross-sections of some possible bubble configurations.

In Section 5 we describe the algorithm used in a series of computations which show that torus bubbles are not minimizers for the two equal volume isoperimetric problem in $\mathbb{R}^{3}$.

Techniques extending those developed in this paper also prove Conjecture 3 for other volume ratios, but at this time do not seem to suffice to cover all cases.

\section{Existence and regularity}

Almgren showed in [3] that there exists an area minimizing surface $S\left(v_{1}, v_{2}\right)$ in $\mathbb{R}^{3}$ among the set of surfaces enclosing volumes $v_{1}, v_{2}$. Here surface refers to a generalized notion defined using the methods of geometric measure theory, specifically what Almgren calls $(F, \varepsilon, \delta)$ sets. For our purposes it suffices that this class includes the piecewise-smooth surfaces. 
TheOrem $2.1([3])$. An area minimizing surface $S\left(v_{1}, v_{2}\right)$ exists and is a smooth surface almost everywhere.

The nature of the singularities of $S\left(v_{1}, v_{2}\right)$ was established by Taylor.

TheOREM 2.2 ([39]). $S\left(v_{1}, v_{2}\right)$ is a piecewise-smooth surface. Its singularities consist of smooth triple curves along which three smooth surfaces come together at an angle of $120^{\circ}$ and isolated points where four triple curves and six pieces of surface converge. At these isolated points the asymptotic cone is the cone over the 1-skeleton of a regular tetrahedron.

Thus Taylor's work established that $S\left(v_{1}, v_{2}\right)$, which Almgren's theorem established as a minimizer among a generalized class of surfaces called $(F, \varepsilon, \delta)$ surfaces, is a bubble, in our terminology. It follows that if we can establish the minimality of the double bubble among our class of bubbles then we will also show it minimizes among the more general class of surfaces considered by Almgren. We now establish some properties of minimizing bubbles. Lemma 2.3, Corollary 2.4 and Lemma 2.5, are standard results in variational geometry. We include brief proofs for completeness. Lemma 2.3 states that the sum of the (oriented) mean curvatures of all points crossed by a path starting and ending in the same region is zero. It implies that the sum of the (oriented) mean curvatures of all points crossed by any path depend only on its starting and ending points. These results hold even if the path crosses through many surfaces, and if it starts and ends in different components of the same region.

LEMMA 2.3. Let $\gamma$ be an oriented curve in $\mathbb{R}^{3}$ intersecting a minimizing bubble $B$ transversely at regular points such that the initial and final points of $\gamma$ lie in the interior of the same region. Then the sum of the mean curvatures of all the points of $\gamma \cap B$, oriented by $\gamma$, is zero.

Proof. Perturb the curve slightly so that each of its intersections with $B$ becomes perpendicular. Consider a deformation of $B$ which pushes points in $\mathbb{R}^{3}$ near $\gamma$ a uniform distance along the curve. To first order this preserves the volume of each region. The derivative of the area, to first order, is given by the sum of the mean curvatures over the points of $\gamma \cap B$. If this sum is nonzero, a deformation can be defined which decreases area while preserving the volume of each region.

A special case of the above lemma occurs when $\gamma$ is a simple closed curve encircling a triple curve of the bubble. The lemma then implies that the sum of the mean curvatures around the triple curve adds up to zero. This local minimization condition is built into our definition of a bubble. More generally, the lemma implies that any two surfaces separating the same pair of regions have the same mean curvature. 
Corollary 2.4. If $S_{1}$ and $S_{2}$ are surfaces in a bubble, each of which separate regions $R_{1}$ and $R_{2}$, then their mean curvatures are equal.

Proof. Consider an arc $\alpha$ which starts in $R_{1}$ passes through $S_{1}$ into $R_{2}$, then transversely through the bubble and on through $S_{2}$ back into $R_{1}$. A subarc $\beta$ of $\alpha$ starts and ends in $R_{2}$. Applying Lemma 2.3 to both $\alpha$ and $\beta$ implies the corollary.

\section{Lemma 2.5. A minimizing bubble $B$ is connected.}

Proof. If not, we can move a component by an isometry of $\mathbb{R}^{3}$ until it touches a distinct component. The resulting singularity violates those allowed in Theorem 2.2.

The following result was first observed by B. White and F. Morgan, and first written down by Foisy in [16]. Since we will need to refer to the proof, we present an argument for the case of two regions in $\mathbb{R}^{3}$. More detailed and general arguments can be found in the work of Hutchings [22] and Morgan [32].

THEOREM 2.6. $S\left(v_{1}, v_{2}\right)$ is a surface of revolution.

Proof. Since $S\left(v_{1}, v_{2}\right)$ is a minimizing bubble, each of its faces separates a pair of distinct regions - either separating $R_{1}$ from $R_{2}$ or one of them from $R_{0}$ (which is not connected if there are empty regions.)

Given any unit vector $Z$ in $\mathbb{R}^{3}$, there is a plane $P_{Z}$ in $\mathbb{R}^{3}$ perpendicular to $Z$ which bisects the total volume $v_{1}+v_{2}$ enclosed by $S\left(v_{1}, v_{2}\right)$. If $Q_{Z}$ is a parallel plane which also bisects the total volume, then $P_{Z}$ and $Q_{Z}$ split the regions $R_{1}$ and $R_{2}$ in the same proportions. (In fact $P_{Z}$ must coincide with $Q_{Z}$, but we do not need to show this. It is also irrelevant to this argument whether $S\left(v_{1}, v_{2}\right)$ contains empty regions.) Let $f(Z)$ denote the proportion of the volume of $R_{1}$ on the side of $P_{Z}$ to which $Z$ points. The function $f(Z)$ is well defined, continuous on the unit 2 -sphere, $0 \leq f(Z) \leq 1$ and $f(-Z)=1-f(Z)$. Along any great circle of directions on the unit 2-sphere, the Intermediate Value Theorem implies that there are at least two points where $f(Z)=1 / 2$ and the plane $P_{Z}$ bisects the volume of both regions. We fix $Z$ to be such a vector.

Consider the intersection of $S\left(v_{1}, v_{2}\right)$ with each of the two half-spaces determined by $P_{Z}$. The intersection of a face of $S\left(v_{1}, v_{2}\right)$ with the plane $P_{Z}$ is given by a graph in $P_{Z}$ with smooth edges and isolated vertices, or else the face is completely contained in $P_{Z}$, since the face is a smooth constant mean curvature surface [18]. Reflection of the smaller area piece of $S\left(v_{1}, v_{2}\right)$ lying in one of these half-spaces gives a new surface $S_{1}\left(v_{1}, v_{2}\right)$ which encloses regions of the same volumes. Since $S_{1}\left(v_{1}, v_{2}\right)$ cannot have less area, it must have the same area as $S\left(v_{1}, v_{2}\right) . S_{1}\left(v_{1}, v_{2}\right)$ has the property that reflection through $P_{Z}$ 
preserves the new regions, which we continue to call $R_{0}, R_{1}$ and $R_{2}$. If the plane $P_{Z}$ contained a face of $S\left(v_{1}, v_{2}\right)$ then this face would now separate two components of the same region in $S_{1}\left(v_{1}, v_{2}\right)$, and area could be reduced by removing that face from $S_{1}\left(v_{1}, v_{2}\right)$, preserving the volume of each region. So $P_{Z}$ cannot contain a face of $S\left(v_{1}, v_{2}\right)$.

We repeat the above argument along a great circle of the unit 2-sphere consisting of the unit vectors which are perpendicular to $Z$, to find a plane $P_{W}$ perpendicular to $P_{Z}$ which bisects the volume of the regions $R_{1}$ and $R_{2}$ in $S_{1}\left(v_{1}, v_{2}\right)$. This process constructs a surface $S_{2}\left(v_{1}, v_{2}\right)$, still having the same area as $S\left(v_{1}, v_{2}\right)$, enclosing regions of the same volumes, and for which reflection through each of $P_{Z}$ and $P_{W}$ preserves $R_{1}$ and $R_{2}$.

Since composing reflections through two perpendicular planes gives a rotation of angle $180^{\circ}$, it follows that rotation of angle $180^{\circ}$ about $L=P_{Z} \cap P_{W}$ preserves the regions $R_{1}$ and $R_{2}$. Now consider any plane $Q$ containing $L$. Note that half of the volume of $R_{1}$ and $R_{2}$ lies on each side of $Q$. As before the plane $Q$ cannot contain a face of $S_{2}\left(v_{1}, v_{2}\right)$. If the intersection of $Q$ with $S_{2}\left(v_{1}, v_{2}\right)$ is not perpendicular to $Q$, then replacing half of $S_{2}\left(v_{1}, v_{2}\right)$ with its reflected image through $Q$ gives a new minimizing surface which is not smooth, and has singularities not allowed by Theorem 2.2. Thus $S_{2}\left(v_{1}, v_{2}\right)$ is perpendicular to each plane through $L$ and is therefore a surface of revolution around $L$. $S_{1}\left(v_{1}, v_{2}\right)$ and $S_{2}\left(v_{1}, v_{2}\right)$ may not a priori be identical, but they coincide on a half-space of $\mathbb{R}^{3}$. Moreover this half-space was chosen arbitrarily, so that $S_{1}\left(v_{1}, v_{2}\right)$ is cut by $P_{W}$ into two pieces, each half of some surface of revolution. The axis of each of these surfaces of revolution is $L=P_{Z} \cap P_{W}$, so they coincide and $S_{1}\left(v_{1}, v_{2}\right)$ itself is a surface of revolution. Similar reasoning shows that $S\left(v_{1}, v_{2}\right)$ is a surface of revolution, and coincides with $S_{2}\left(v_{1}, v_{2}\right)$ everywhere. If we make $S_{1}$ by reflecting the other half of $S$ across $P_{Z}$, then the same argument shows that the other half of $S$ is also a surface of revolution about a line $L^{\prime}$ in $P_{Z}$. The Almgren and Taylor regularity results, and in particular the unique continuation property of constant mean curvature surfaces, imply that $L^{\prime}=L$, so $S$ itself is a surface of revolution about $L$.

We next summarize some key results obtained by Hutchings in [22]. Recall that $S\left(v_{1}, v_{2}\right)$ is a minimizing bubble that separates $\mathbb{R}^{3}$ into regions $R_{1}$ of volume $v_{1}$ and $R_{2}$ of volume $v_{2}$ and that $a\left(v_{1}, v_{2}\right)$ is the area of $S\left(v_{1}, v_{2}\right)$.

TheOREM 2.7. The function $a\left(v_{1}, v_{2}\right)$ is strictly concave on $[0,+\infty) \times$ $[0,+\infty)$.

Corollary 2.8. If $v_{1}^{\prime}>v_{1}$ then $a\left(v_{1}^{\prime}, v_{2}\right)>a\left(v_{1}, v_{2}\right)$.

Proof. As $v_{1} \rightarrow+\infty, a\left(v_{1}, v_{2}\right) \geq a\left(v_{1}\right) \rightarrow+\infty$. Concavity of the function $a$ implies the corollary. 
Corollary 2.9. $S\left(v_{1}, v_{2}\right)$ has no empty regions.

Proof. If there is an empty region, join it it to one of the other regions by removing a face on its boundary and apply the previous corollary.

Deeper connectedness results also follow from concavity. In particular, Hutchings deduced that each of $R_{1}$ and $R_{2}$ is connected if the volumes are equal.

TheOrem 2.10. $S\left(v_{1}, v_{1}\right)$ encloses exactly two connected components.

Proof. See Hutchings [22, Th. 4.2].

\section{Delaunay surfaces}

In this section, we summarize the classification theory of constant mean curvature surfaces of revolution, the Delaunay surfaces, and present some properties of these surfaces that will be used in our study of bubbles.

The mean curvature of a surface in $\mathbb{R}^{3}$ is the trace of the second fundamental form of the surface. Computing this requires choosing a unit normal vector field to the surface. The mean curvature vector field, formed by scaling a unit normal vector field by the mean curvature, gives the direction of a variation which decreases the area of a surface as quickly as possible [38].

For a surface of revolution we have simple formulas for the mean curvature. Consider a surface of revolution about the $x$-axis in $\mathbb{R}^{3}$, with generating curve contained in the upper half-plane. If the generating curve is a graph, $y=y(x)$, then the mean curvature $h$, equal to the sum of the principal curvatures, is given by the formulas:

$$
\begin{gathered}
k_{m}=\frac{-\ddot{y}}{\left(1+\dot{y}^{2}\right)^{3 / 2}}, \\
k_{p}=\frac{1}{y \sqrt{1+\dot{y}^{2}}}, \\
h=k_{m}+k_{p},
\end{gathered}
$$

where $k_{m}$ is the curvature at $(x, y)$ of the generating curve in the $x y$-plane (sometimes called the meridian, or profile curve) and $k_{p}$ is the normal curvature of the parallel curve. The value of $k_{p}$ is equal to the reciprocal of the distance to the $x$-axis along the perpendicular to the generating curve [38]. Note that the mean curvature of a unit sphere equals two. For surfaces of revolution generated by curves which are not graphs, we need to specify an orientation on the generating curve to fix the sign of the mean curvature. We

use the convention that the sign of the mean curvature is given by the formulas 
above if the curve is oriented left-to-right, or equivalently if the graph's tangent vector has a positive $x$-component. If its tangent vector has a negative $x$-component, the signs in the formulas for $k_{m}$ and $k_{p}$ should be reversed in the above formulas. For curves with vertical tangent vectors the above formulas do not apply directly, but in our applications vertical tangents are isolated points, and the limits of the above formulas will give $k_{m}$ and $k_{p}$ at vertical tangencies.

Surfaces of revolution having constant mean curvature were first studied by Euler, and classified by Delaunay [13]. The term Delaunay surface is used to refer to these surfaces.

THEOREM 3.1. The surfaces of revolution of constant mean curvature are the plane, sphere, catenoid, cylinder, unduloid, and nodoid. The generating curves of a nodoid and an unduloid, called the nodary and the undulary, are periodic along the $x$-axis, and have exactly one local minimum and one local maximum in each period. The undulary is a graph over the $x$-axis. The nodary has one local maximum, one local minimum and two vertical tangencies in each period.

Proof. Expositions of the classification of Delaunay surfaces can be found in [14] and [23].

The nodoid and unduloid are not as well known as the other surfaces. An undulary can be obtained by rolling an ellipse along the $x$-axis and tracing the path taken by one of the foci. A nodary can be obtained by rolling a hyperbola [14]. See Figure 6.
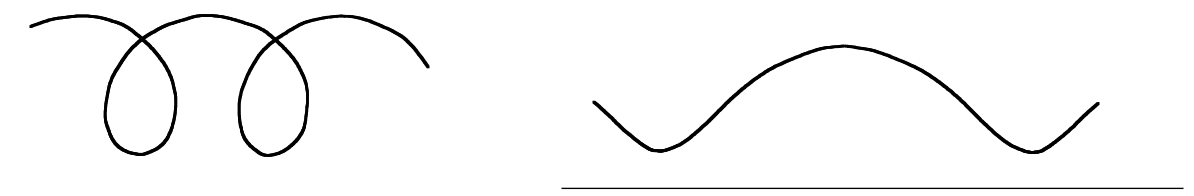

Figure 6. Portions of a nodary and an undulary, generating curves of a nodoid and an unduloid.

With their generating curves oriented left to right, the mean curvature of each of the Delaunay surfaces is nonnegative, with the exception of the nodoid. The sign of the mean curvature of a nodoid depends upon the choice of orientation for its generating nodary, and can be positive or negative.

If a surface of revolution minimizes area among all surfaces of revolution surrounding a given volume, then its generating curve satisfies an associated Euler-Lagrange equation. This second order ODE implies that the mean curvature is constant [14]. 
The mean curvature of the surface of revolution generated by a $C^{2}$ graph $y(x)$ is given by:

$$
h=k_{m}+k_{p}=\frac{-\ddot{y}}{\left(1+\dot{y}^{2}\right)^{3 / 2}}+\frac{1}{y \sqrt{1+\dot{y}^{2}}} .
$$

We can integrate this ODE once, after multiplying through by $2 y \dot{y}$.

$$
\begin{aligned}
0 & =h+\frac{\ddot{y}}{\left(1+\dot{y}^{2}\right)^{3 / 2}}-\frac{1}{y \sqrt{1+\dot{y}^{2}}} \\
& =2 h y \dot{y}+\frac{2 y \dot{y} \ddot{y}}{\left(1+\dot{y}^{2}\right)^{3 / 2}}-\frac{2 \dot{y}}{\sqrt{1+\dot{y}^{2}}} \\
& =\frac{d f(x)}{d x}
\end{aligned}
$$

where $f(x)$ is the function

$$
f(x)=h y^{2}-\frac{2 y}{\sqrt{1+\dot{y}^{2}}} .
$$

Therefore $f$ is constant along the graph. The fact that the equation for constant mean curvature surfaces of revolution has a first integral was known to Plateau [35, pp. 138-139], who in turn references work of Beer. Korevaar, Kusner and Solomon called $f$ the force of a constant mean curvature surface, and pointed out that it has a physical interpretation as the net force exerted by a soap film on a plane cutting off an end of the surface. They also showed that it could be defined for more general constant mean curvature surfaces [25], where it serves as a useful analytical tool.

A convenient way of expressing the force of a constant mean curvature surface of revolution which holds even when the generating curve is not a graph is given by the formula

$$
f=h y^{2}-2 y \cos \alpha .
$$

Here $\alpha$ denotes the angle between the positive $x$-axis and the generating curve of the surface of revolution, which is an oriented curve. This makes sense for all values of $\alpha$. In our applications, we alway orient the generating curve of a Delaunay surface, and thus fix an unambiguous sign for the mean curvature.

Lemma 3.2. Given $h, x_{0}, y_{0}>0$ and $\dot{y_{0}}$, there exists a unique Delaunay curve $\delta$ through the point $\left(x_{0}, y_{0}\right)$ having slope $\dot{y}_{0}$ and generating a Delaunay surface $D$ having mean curvature $h$. The slope $\dot{y}_{0}$ can be infinite. The Delaunay surface is a sphere $S$ if and only if

$$
h=h_{S}=\frac{2}{y_{0} \sqrt{1+\dot{y}_{0}^{2}}} .
$$


If $h>h_{S}$, then $D$ is a nodoid and $\delta$ lies strictly below the circle $\sigma$ generating $S$ in a neighborhood of $\left(x_{0}, y_{0}\right)$. An arc of $\delta$ which is decreasing as it leaves $\left(x_{0}, y_{0}\right)$ remains beneath $\sigma$ as long as it remains a graph over the $x$-axis.

If $h<h_{S}$, $\delta$ lies strictly above $\sigma$ in a neighborhood of $\left(x_{0}, y_{0}\right)$. If $0<h<$ $h_{S}$ then $D$ is an unduloid or cylinder, if $h<0$ then $D$ is a nodoid, and if $h=0$ then $D$ is a catenoid.

Proof. Solving equation 1 for $\ddot{y}$ gives a second order ODE satisfied by the generating curves of Delaunay surfaces at points where $\dot{y}$ is finite:

$$
\ddot{y}=-h\left(1+\dot{y}^{2}\right)^{3 / 2}+\frac{1+\dot{y}^{2}}{y} .
$$

Given a constant $h$ and a choice of initial conditions $y$ and $\dot{y}$ this equation has a unique solution from standard existence and uniqueness of solutions to ODEs. These solutions can also be obtained by rolling an appropriate ellipse, hyperbola or parabola [14]. The latter approach also shows that we can find a nodoid passing through $y$ with any mean curvature $h \neq 0$ in the case where $\dot{y}$ is infinite.

The mean curvature of the unique sphere centered on the $x$-axis and having slope $\dot{y}_{0}$ at $\left(x_{0}, y_{0}\right)$ is given by $h_{S}$. Assume now that $\sigma$ is centered at $(0,0)$ on the $x y$-plane.

If $h>h_{S}$ then Equation 5 implies that $\delta$ lies strictly below $\sigma$ in a neighborhood of $\left(x_{0}, y_{0}\right)$. If $D$ is an unduloid then $\delta$ is an undulary whose graph crosses $\sigma$ again in at least two more points $\left(x_{1}, y_{1}\right)$ and $\left(x_{2}, y_{2}\right)$. We can assume, after performing a left/right reflection if necessary, that $x_{0} \geq 0$ and $x_{1}>x_{0}$. Denote by $\gamma$ the subcurve of $\delta$ starting at $\left(x_{0}, y_{0}\right)$ and running to $\left(x_{1}, y_{1}\right)$. Let $\gamma_{t}=\gamma+(t, 0)$ be a horizontal translate of $\gamma$ by a distance $t$ and let $T=\sup \left\{t: \gamma_{t} \cap S \neq \emptyset\right\}$. Then $\gamma_{T}$ is tangent to $S$ at a point $P$ and lies above $\sigma$ in a neighborhood of $P$. This violates Equation 5, so $D$ cannot be an unduloid. $D$ cannot be a cylinder or a catenoid, so it can only be a nodoid. If a subarc $\gamma$ of $\delta$ which is decreasing as it leaves $\left(x_{0}, y_{0}\right)$ intersects $\sigma$ while $\delta$ remains a graph then an identical argument shows that we can horizontally translate $\gamma$ until it is tangent to $\sigma$ while lying above it, which is impossible by equation 5. (In fact it will remain beneath $\sigma$ for somewhat longer than this, but this will not be relevant for us.)

If $0<h<h_{S}$ then equation 5 implies that $\delta$ lies strictly above $\sigma$ in a neighborhood of $\left(x_{0}, y_{0}\right)$. If $D$ is a nodoid, consider the subarc $\beta$ of $\delta$ decreasing from $\left(x_{0}, y_{0}\right)$ until it reaches a vertical tangency $\left(x_{v}, y_{v}\right)$. The arc $\beta$ is a graph which may or may not cross $\sigma$ at an additional point. In either case we can translate $\beta$ horizontally to the left until the last time at which it intersects $\sigma$. The final intersection must be at an interior point of $\beta$, so after translation 
$\beta$ becomes tangent to $\sigma$ at an interior point while lying underneath $\sigma$, again impossible by equation 5 . Thus the surface must be an unduloid or cylinder.

If $h<0$ then the surface must be a nodoid.

Nodoids and unduloids can be distinguished by the sign of the product $f h$.

Corollary 3.3. For a nodoid, $f h>0$. For an unduloid and a cylinder $f h<0$. For a sphere, a catenoid and a plane, $f h=0$.

Proof. For a sphere of radius $r$, we can compute $f$ at a maximum point where

$$
f=h r^{2}-2 r .
$$

Since $h=2 / r$, we have $f=0$. We also compute $f h$ for an unduloid or cylinder at a local maximum. By Lemma 3.2 we know that $0<h<h_{S}=2 / r$. Then

$$
f=h r^{2}-2 r<(2 / r) r^{2}-2 r=0 .
$$

So $h>0$ and $f<0$ implying that $h f<0$.

We compute $f h$ for a nodoid at a vertical tangency, where $\dot{y}=+\infty$ and $f=h y^{2}$. Then $f h=h^{2} y^{2}>0$.

The next proposition deduces some useful properties of Delaunay surfaces.

Proposition 3.4. Let $D$ be a Delaunay surface with generating curve $\delta$, mean curvature $h$, maximum $y$-value $y_{M}$ and minimum $y$-value $y_{m}$. Then

1. If $\delta$ is a nodary or undulary, the arc-length of one period of $\delta$ is $2 \pi / h$.

2. If $\delta$ is an undulary, the period $P$ of $\delta$ satisfies

$$
2\left(y_{M}+y_{m}\right) \leq P \leq \pi\left(y_{M}+y_{m}\right) .
$$

3. If $\delta$ is a nodary,

$$
h=2 /\left(y_{M}-y_{m}\right) .
$$

If $\delta$ is an undulary,

$$
h=2 /\left(y_{M}+y_{m}\right) .
$$

4. If $\delta$ is a nodary, $\delta$ has nonzero curvature.

Proof.

1. In [23] the equation for the generating curve of a Delaunay surface is given in terms of arclength, and it is shown that nodaries and undularies are periodic, with the arc length of one period given by $2 \pi / h$. 
2. The period of an undulary is equal to the perimeter of the ellipse that is rolled along the $x$-axis to generate it [14]. The inequalities follow by comparing twice the length of the major axis, $2\left(y_{M}+y_{m}\right)$, with the perimeter of an enclosing circle whose diameter is the length of the major axis, $\pi\left(y_{M}+y_{m}\right)$.

3. If $\delta$ is a nodary, we calculate the force at $y_{M}$ and $y_{m}$. At $y_{M}$ we get

$$
f=h y_{M}^{2}-2 y_{M} \text {. }
$$

At $y_{m}$ we get

$$
f=h y_{m}^{2}+2 y_{m},
$$

where the sign of the second term changes since the orientation has reversed. Setting these equal we get

$$
h=\frac{2}{y_{M}-y_{m}} .
$$

For an unduloid, the proof is identical except for one sign change.

4. At a point where $\delta$ has zero curvature, $k_{m}=0$ so

$$
h=k_{p}=\frac{1}{y \sqrt{1+\dot{y}^{2}}} .
$$

Equation 4 gives the mean curvature of a sphere with slope $\dot{y}$ at height $y$ as:

$$
h_{S}=\frac{2}{y \sqrt{1+\dot{y}^{2}}}>0 .
$$

Where $\dot{y}$ is defined, this is exactly twice the value of $h$ calculated above. For a nodary, $h>h_{S}$ or $h<0$ by Lemma 3.2, so we cannot have $h_{S}=2 h$ and there are no points of zero curvature on a nodary. At points where the nodary is vertical, $h=k_{m}$ since $k_{p}=0$. Thus the curvature is nonzero at vertical tangencies except possibly when $h=0$, which would imply that the Delaunay surface is a plane or a catenoid, rather than a nodoid as assumed.

Important stability formulas for constant mean curvature surfaces were developed by J. L. Barbosa and M. do Carmo [8]. Stability refers to the behavior of a surface when a compact subsurface is deformed by a variation, while holding its boundary fixed. A subsurface of a complete constant mean curvature surface is called stable if there is no compactly supported normal variation which decreases its area while preserving the volumes on each side, and unstable otherwise. Unstable constant mean curvature surfaces cannot form part of a minimizing bubble. 
Proposition 3.5. The smooth subsurfaces of a minimizing bubble $S\left(v_{1}, v_{2}\right)$ are stable subsurfaces of Delaunay surfaces.

Proof. $S\left(v_{1}, v_{2}\right)$ is a piecewise-smooth surface of revolution whose smooth subsurfaces have constant mean curvature by Theorems 2.6 and 2.2. Any constant mean curvature surface of revolution in $\mathbb{R}^{3}$ is a Delaunay surface. If one of these subsurfaces is unstable, then there is a variation which maintains the volume of each region while decreasing the area. The surface then cannot be part of a minimizing bubble.

We next deduce a formula for the horizontal distance between two points on a Delaunay curve whose $y$-coordinates are known. We require that the second point be above the first, but it can be either to the left or to the right. We also get a formula for the volume under a Delaunay surface.

Proposition 3.6. Let $\left(x_{1}, y_{1}\right)$ and $\left(x_{2}, y_{2}\right)$ be two points on a Delaunay curve. Suppose that $y_{1}<y_{2}$ and that between the points, $\frac{d y}{d x} \neq 0$. Then

$$
x_{2}-x_{1}=\int_{y_{1}}^{y_{2}} \frac{t}{\sqrt{(2 y+t)(2 y-t)}} d y
$$

where $t=t(y)=h y^{2}-f$.

$$
\text { If } x_{1}<x_{2} \text { and the curve also satisfies } \frac{d x}{d y}>0 \text { between the two points then }
$$

the volume underneath the surface of revolution generated by the curve between $x=x_{1}$ and $x=x_{2}$ is given by:

$$
V\left(x_{1}, x_{2}\right)=\int_{y_{1}}^{y_{2}} \frac{\pi y^{2} t}{\sqrt{(2 y+t)(2 y-t)}} d y .
$$

If instead we have $x_{2} \leq x_{1}$ in the previous case then the formula gives the negative of the volume underneath the surface of revolution generated by the curve between $x=x_{1}$ and $x=x_{2}$.

Proof. We solve equation 2 for $d x / d y$, using that $d y / d x \neq 0$, and then apply the change of variables formula.

$$
\begin{aligned}
f & =h y^{2}-\frac{2 y}{\sqrt{1+\dot{y}^{2}}} \\
\sqrt{1+\dot{y}^{2}} & =\frac{2 y}{t} \\
\frac{d x}{d y} & =\frac{t}{\sqrt{(2 y+t)(2 y-t)}}
\end{aligned}
$$


Note that the sign of $t=t(y)=2 y \cos \alpha$ is positive if and only if the curve is oriented to the right, i.e. if $\cos \alpha>0$ in equation 3. Hence the integrand gives the correctly signed measure of displacement in the $x$ direction.

The volume of the region enclosed by a surface of revolution generated by a graph $y(x)$ between $x_{1}, x_{2}$ with $x_{1} \leq x_{2}$ is given by

$$
v=\int_{x_{1}}^{x_{2}} \pi y^{2} d x
$$

Since we have already deduced a formula for $d x$, the volume formulas follow immediately.

Note that if $y_{1}>y_{2}$ then the formulas in Proposition 3.6 would have the sign reversed. A choice in sign was made in taking the positive square root, and this choice is correct if the curve is oriented so that $y$ is increasing. However we need to assume that $x_{2}>x_{1}$ only when calculating the volumes, and not in equation 6.

The integrals of Proposition 3.6 are singular at a local minimum or maximum, where $d y / d x=0$, and we need to apply a change of variables to obtain a formula which holds near such points. Note that the formulas below do not require that $y_{2}>y_{1}$, but do require that the Delaunay curve is a graph over the $x$-axis, with $x_{2}>x_{1}$.

Proposition 3.7. Let $\left(x_{1}, y_{1}\right)$ and $\left(x_{2}, y_{2}\right)$ be two points on a Delaunay curve which is a graph over the $x$-axis with $x_{2}>x_{1}$ and suppose there is exactly one critical point between the two points, with $y$-value labeled $y_{\min }$ or $y_{\max }$ depending on whether it is a local minimum or maximum. Let $h, f$ be the mean curvature and force associated to the Delaunay surface generated by the curve. Set $t=t(y(z))=h(y(z))^{2}-f$.

If $y_{\min }$ is a local minimum, let $y=y(z)=y_{\min }+z^{2}, z_{1}=-\sqrt{y_{1}-y_{\min }}$ and $z_{3}=\sqrt{y_{2}-y_{\min }}$. Then

$$
x_{2}-x_{1}=\int_{z_{1}}^{z_{3}} \frac{2 t}{\sqrt{(2 y+t)\left(2-h y_{\min }-h y\right)}} d z
$$

and

$$
V\left(x_{1}, x_{2}\right)=\int_{z_{1}}^{z_{3}} \frac{2 \pi y^{2} t}{\sqrt{(2 y+t)\left(2-h y_{\min }-h y\right)}} d z .
$$

If $y_{\max }$ is a local maximum, let $y(z)=y_{\max }-z^{2}, z_{2}=\sqrt{y_{\max }-y_{2}}$ and $z_{4}=-\sqrt{y_{\max }-y_{1}}$. Then

$$
x_{2}-x_{1}=\int_{z_{4}}^{z_{2}} \frac{2 t}{\sqrt{(2 y+t)\left(h y-2+h y_{\max }\right)}} d z
$$

and

$$
V\left(x_{1}, x_{2}\right)=\int_{z_{4}}^{z_{2}} \frac{2 \pi y^{2} t}{\sqrt{(2 y+t)\left(h y-2+h y_{\max }\right)}} d z .
$$


Finally, if the curve is an increasing graph over the $x$-axis between $x_{1}$ and $x_{2}$ then equation 8 continues to hold if the sign of $z_{1}$ is changed.

Proof. These formulas follow from applying a change of variables to $y$ in equations 6 and 7 . Suppose $y_{\min }$ is a local minimum. Divide the Delaunay curve into the two pieces on each side of the minimum, and apply Proposition 3.6 and the substitution $y=y_{\min }+z^{2}$ to each piece.

One of the terms in the square root in the denominator in equation 6 factors as

$$
2 y-t=2 y-h y^{2}+f=\left(y-y_{\min }\right)\left(2-h y_{\min }-h y\right)=z^{2}\left(2-h y_{\min }-h y\right)
$$

since $f=h y_{\min }^{2}-2 y_{\min }$. A resulting factor of $z$ in the denominator, which causes the singularity, cancels with a $z$ in the numerator from the change of variables. The integrands are even functions of $z$, and $y=y_{\min }$ corresponds to $z=0$, so the curve portions on opposite sides of the minimum can be mapped to positive and negative $z$ values, and combined into one integral.

The maximum case is similar. Near a maximum the substitution $y=$ $y_{\max }-z^{2}$ is used. Several minus signs appear in the derivation, resulting in the given expression.

The final statement in the proposition is a direct application of the change of variables formula to equation 6 .

\section{Torus bubbles}

We established in Lemma 3.5 that a minimizing bubble must be obtained by revolving a union of Delaunay curves contained in the upper half plane. A key case of such a bubble is the torus bubble, constructed as follows. Take two circular arcs of the same radius, facing each other, each with one endpoint and center on the $x$-axis, and connect the other endpoints with two different Delaunay curves meeting at 120 degrees. Rotating around the $x$-axis, we get a piecewise-smooth surface surrounding two components, one homeomorphic to a torus, which we call the torus component $T$, and one homeomorphic to a ball, which we call the ball component $B$. It is not immediately clear whether it is possible to make such a construction so that the curves meet at $120^{\circ}$ angles and the mean curvatures sum to zero around each triple curve. Such torus bubbles do indeed exist, and we will need to show that none of them are minimizers. Figure 7, due to J. Sullivan, shows a torus bubble.

The reason that torus bubbles play so central a role in our argument is due to another key result of Hutchings:

TheOREM 4.1. A minimizing bubble enclosing two equal volumes must be either a double bubble or a torus bubble. 


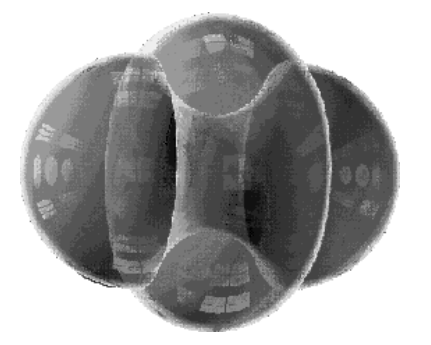

Figure 7. A torus bubble in $\mathbb{R}^{3}$.

Proof. Theorem 2.10 states that $S(v, v)$ contains two connected components. The two possible configurations are then a consequence of Hutchings' structure theorem for minimizing bubbles [22, Th. 5.1].

We label the surfaces of a minimizing torus bubble as indicated in Figure 8. The component homeomorphic to a solid torus is denoted $T$ and the component homeomorphic to a ball is denoted $B$. The volumes of the inner ball component and outer torus component are denoted by $v_{i}$ and $v_{o}$ respectively. The inner Delaunay surface on the boundary of $T$ is denoted $T_{i}$ and the outer Delaunay surface is denoted $T_{o}$. The generating curve of $T_{o}$ is denoted by $\tau_{o}$ and that of $T_{i}$ by $\tau_{i}$. The angles subtended by the generating curves for $S_{1}$ and $S_{2}$ are denoted by $\theta_{1}$ and $\theta_{2}$. The mean curvatures of $T_{i}$ and $T_{o}$ are $h_{i}$ and $h_{o}$, with signs chosen so that so that $h_{i}$ is positive if the mean curvature vector points into $B$ and negative if it points into $T$, and $h_{o}$ is positive if the mean curvature vector points into $T$ and negative if it points towards outside the bubble.

Denote by $\left(x_{1}, y_{1}\right)$ the initial point where the two Delaunay surfaces start, and $\left(x_{2}, y_{2}\right)$ the point where they rejoin. To specify a sign for the mean curvature, we orient the generating curves $\tau_{i}$ and $\tau_{o}$ of $T_{i}$ and $T_{o}$ so that they run from $\left(x_{1}, y_{1}\right)$ to $\left(x_{2}, y_{2}\right)$.

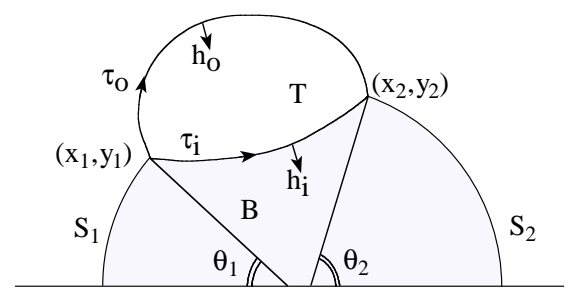

Figure 8. Parameters of a torus bubble.

Torus bubbles for various ranges of $\theta_{1}$ are indicated in Figures 18, 19 and 20. We will show that $\theta_{1}$ and $h_{o}$ parameterize the space of torus bubbles and that no choice of $\theta_{1}$ and $h_{o}$ gives a minimizing bubble. To do so, we first 
present some properties of minimizing torus bubbles, mainly derived from the work of Hutchings.

Lemma 4.2. In a minimizing torus bubble, $S_{1}$ and $S_{2}$ have the same mean curvature.

LEMmA 4.3. In a minimizing torus bubble, $h_{o} \geq 0$ and $h_{i}=2-h_{o}$.

Proof. The three mean curvatures around a triple curve sum to zero by Lemma 2.3 implying that $h_{o}=2-h_{i}$. If $h_{o}<0$ then there is a deformation which pushes $T_{o}$ outward, increasing the volume of the torus component and decreasing its area. Corollary 2.8 then gives a contradiction.

Proposition 4.4. The regions bounded by a minimizing torus bubble are not all preserved by reflection through some plane $P$ perpendicular to the $x$-axis.

Proof. If there is such a symmetry, then the bubble is invariant by reflection through the $x y$-plane and by reflection through a perpendicular plane $P$. The argument given in Theorem 2.6 shows that the bubble is a surface of revolution around the line of intersection of these two planes. But it is also a surface of revolution around the $x$-axis, and therefore a surface of revolution around two perpendicular axis. It must then be a union of spheres, and not a torus bubble.

COROLlary 4.5. Given a minimizing equal-volume torus bubble, we can rescale and reflect so that $\theta_{1}<\theta_{2}$ and each of the two circular arcs generating $S_{1}$ and $S_{2}$ has curvature one.

Proof. If $\theta_{1}=\theta_{2}$, Lemma 4.4 gives a contradiction. If $\theta_{1}>\theta_{2}$, a left $/$ right reflection interchanges the two angles. Lemma 4.2 implies that the two circular arcs generating $S_{1}$ and $S_{2}$ have the same curvature, and thus the same radius. We can rescale and assume without loss of generality that each has radius one (and thus mean curvature two).

From now on we will restrict attention to torus bubbles with $\theta_{1}<\theta_{2}$ and with spherical caps of radius one.

Proposition 4.6. If a torus bubble contains a surface $T_{1}$ which is a subsurface of a nodoid and the nodary generating $T_{1}$ contains two interior vertical tangencies, then $T_{1}$ is unstable and not a part of a minimizing bubble.

Proof. We will construct a volume preserving Jacobi field supported on a proper subset of $T_{1}$, establishing instability as in [8, Prop. 2.24]. 
We start with the constant unit vector field $v$ pointing in the direction of the positive $y$-axis. The vector field $v$ is tangent to $T_{1}$ along two circles which are generated by rotating the interior vertical tangencies of the nodary. Let $T_{1}^{\prime}$ denote the compact portion of $T_{1}$ between these two circles. Define the vector field $W=\langle v, N\rangle N$. where $N$ is the normal vector field along $T_{1}^{\prime}$. and extend $W$ to be the zero vector field elsewhere on $T_{1}$. The integral of the function $f=\langle v, N\rangle$ along $T_{1}$ is zero, since the involution $(x, y) \rightarrow(-x,-y)$ of $T_{1}$ takes $f$ to $-f$. So the normal variation defined by $f$ preserves volume in the sense of [8, Prop. 2.24]. Moreover [8, Prop. 2.24] shows that $f N$ is a Jacobi field, since it is constructed by taking the normal part of a constant vector field, and since the nodary extends beyond the two vertical tangencies, the corresponding Jacobi field is supported on a proper subset of $T_{1}$. The Morse Index Theorem implies that the surface $T_{1}$ is unstable and that there is a smaller area surface separating the same volumes.

The next proposition establishes that minimizing torus bubbles do not contain Delaunay curves which are longer than a full period.

Proposition 4.7. If $\tau_{i}$ or $\tau_{o}$ contains a full period, then the torus bubble is not a minimizer.

Proof. Suppose to the contrary that a minimizing torus bubble contains a subsurface generated by a curve which contains a full period. If a boundary surface of the torus component is a subsurface of a nodoid with width more than a period, then the associated nodary contains two vertical tangencies, which implies instability by Proposition 4.6. Similarly, if a boundary surface of the torus component is a subsurface of a cylinder of radius $r$ and its width is greater than $2 \pi r$, the period of a cylinder, than the surface is shown to be unstable in [8].

If a boundary surface of the torus component is a subsurface $U$ of an unduloid then the lemma is less obvious. Note that we cannot necessarily construct a volume preserving Jacobi field from the vector field $\partial / \partial x$, since the Delaunay curve may not contain two maxima or two minima. We establish the lemma with a reflection argument. Assume first that the unduloid containing a full period is the inner surface $T_{i}$.

An arc $u$ of an undulary which is longer than a period contains three distinct points with the same $y$ value, $\left(x_{1}, y_{1}\right),\left(x_{2}, y_{1}\right),\left(x_{3}, y_{1}\right)$, with $x_{1}<$ $x_{2}<x_{3}$, and with the slopes at $\left(x_{1}, y_{1}\right),\left(x_{3}, y_{1}\right)$ each being nonzero and equal to the negative of the slope at $\left(x_{2}, y_{1}\right)$. Reflect the part of $u$ between the planes $x=x_{1}$ and $x=x_{3}$ through the plane $x=\left(x_{1}+x_{3}\right) / 2$ to get a new surface of revolution $U^{\prime}$ generated by $u^{\prime}$, the reflection of $u$. Reflection preserves both the area and the volume under $U$ between the planes $x=x_{1}$ and $x=x_{3}$. Suppose 
first that $u^{\prime}$ does not intersect $\tau_{o}$. Then the volume of the torus bubble is also preserved. The reflection creates a surface which is not smooth along the planes $x=x_{1}$ and $x=x_{3}$ since reflection changes the sign of the slope of the reflected surface along these planes, and this slope was not zero. Thus we have found a solution to the minimization problem which contains a surface which contradicts Theorem 2.2.

Possibly $u^{\prime}$ does intersect the outer curve $\tau_{o}$. See Figure 9 . In that case the total volume enclosed by the union of $U^{\prime}$ and $T_{o}$ between $x=x_{1}$ and $x=x_{3}$ is increased, or at least not decreased. We know that $U^{\prime}$ does not intersect the spherical caps of the torus bubble, since they do not meet the region between the planes $x=x_{1}$ and $x=x_{3}$. Since the volume under $U^{\prime}$ is equal to the volume of the ball region of the torus bubble $B$, the volume in the remaining (disconnected) region must be larger than that in the original torus region $T$. There is no increase in area, contradicting Corollary 2.8.
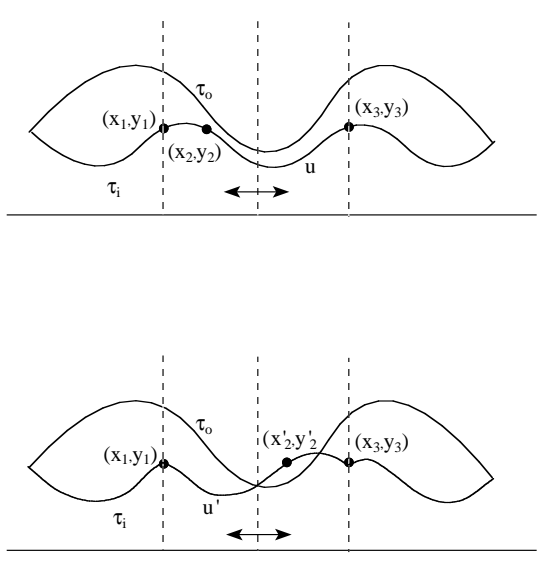

Figure 9. Reflection of a piece of $T_{i}$ could lead to extra intersections.

Note that have used here the fact that two distinct Delaunay curves intersect locally at finitely many points.

Assume now that the unduloid is the outer surface $T_{o}$. We again reflect it to get a new boundary surface $U^{\prime}$. The total volume underneath $U^{\prime}$ is the same as that underneath $U$. Possibly $T_{i}$ intersects the reflected surface $U^{\prime}$. In this case the total volume underneath the union of the two surfaces has increased. Divide the region underneath $U^{\prime}$ so that the region under $T_{i}$ is allocated entirely to $B$, as before, and all other regions are allocated to $T$. Then the total volume of $B$ is preserved, that of $T$ is increased, and the area is unchanged, a contradiction as before.

We note that a somewhat similar argument was used by Athanassenas [7] in studying the stability of minimizing capillary surfaces. See also [41]. Our 
situation has some additional complications due to the possible intersections resulting from the reflection.

LEMMA 4.8. If a torus bubble has $\theta_{1}<60^{\circ}$ and $\theta_{2}<60^{\circ}$ then it is not minimizing. In particular, $\theta_{2} \geq 60^{\circ}$ in a minimizing torus bubble.

Proof. If both $\theta_{1}$ and $\theta_{2}$ are less than $60^{\circ}$, the outer bubble $T_{o}$ is generated by a nodary that becomes vertical twice. Proposition 4.6 implies it is unstable. Since $\theta_{2}$ is the larger angle, $\theta_{2} \geq 60^{\circ}$.

Lemma 4.9. Suppose that $T$ is a torus bubble with $\theta_{2} \geq 120^{\circ}$. The the torus component is completely contained inside the smaller region of the double bubble whose larger outer sphere subtends an angle equal to $\theta_{2}$. Similarly the ball component contains in its interior the larger region of this double bubble.

Proof. Consider a double bubble which is symmetric around the $x$-axis and whose larger spherical cap $S_{2}$ is on the right and subtends an angle of $\theta_{2}$ with the $x$-axis. Denote the smaller (leftmost) spherical cap by $S_{o}$ and the spherical cap forming the interface by $S_{i}$. Then $S_{o}$ is tangent to $\tau_{o}$ at $\left(x_{1}, y_{1}\right)$. We will show that a torus region must be contained inside the smaller component $B_{1}$ of this double bubble, the component bounded by $S_{2}$ and $S_{i}$. Otherwise, we will show that $\tau_{i}$ and $\tau_{o}$ cannot rejoin after leaving $\left(x_{1}, y_{1}\right)$, and thus cannot generate a torus region. See Figures 10 and 8 .

Given a vector in the upper half-plane, there is a unique circle perpendicular to the $x$-axis which is tangent to that vector. A Delaunay curve tangent to the same vector and generating a Delaunay surface with greater mean curvature than the corresponding sphere is a nodary, by Lemma 3.2. A Delaunay curve tangent to the same vector and generating a Delaunay surface with smaller, but still positive, mean curvature is an undulary or horizontal line.

If the mean curvature of $T_{o}$ is equal to that of $S_{o}$, then the mean curvature of $T_{i}$ is equal to that of $S_{i}$ by Lemma 4.3. It follows from Lemma 3.2 that $T_{o}, T_{i}$ and $S_{o}, S_{i}$ coincide, and that there is no torus component.

Suppose now that the mean curvature of $T_{o}$ is smaller than that of $S_{o}$. Then the mean curvature of $T_{i}$ is smaller than that of $S_{i}$ by Lemma 4.3. Since $h_{o} \geq 0$, also by Lemma 4.3 , it follows that $T_{o}$ cannot be a nodoid, and therefore $\tau_{o}$ is a graph over the $x$-axis. In particular, $\tau_{o}$ does not recross the spherical cap $S_{o}$. The generating curve $\tau_{i}$ for $T_{i}$ leaves the point $\left(x_{1}, y_{1}\right)$ on the positive (right) side of $S_{i}$, and lies above $S_{i}$. Since $S_{2}$ forms part of the torus bubble, $\tau_{i}$ must remeet $\tau_{o}$ without crossing $S_{2}$ or itself if it is to generate a torus component with $\tau_{o}$. If it is a graph over the $x$-axis then it cannot remeet $\tau_{o}$ at all. If it is not a graph, then it is a nodary which generates a nodoid with negative mean curvature. Such a curve crosses $S_{2}$ before it can remeet $\tau_{o}$. 
Finally, suppose that the mean curvature of $T_{o}$ is greater than that of $S_{o}$. Then Lemma 4.3 implies that the mean curvature of $T_{i}$ is greater than that of $S_{i}$, and Lemma 3.2 implies that both $T_{o}$ and $T_{i}$ are subsurfaces of nodoids and that near $\left(x_{1}, y_{1}\right), T_{o}$ lies below $S_{o}$ and $T_{i}$ lies below $S_{i}$. See Figure 11 . The nodary $\tau_{o}$ generating $T_{o}$ either crosses itself before leaving $B_{1}$ or leaves $B_{1}$ through $S_{i}$. Similarly, the nodary $\tau_{i}$ generating $T_{i}$ either crosses itself before leaving $B_{1}$ or leaves $B_{1}$ through $S_{o}$. The curves $\tau_{o}$ and $\tau_{i}$ cannot self-intersect before remeeting, since they generate a torus component. Therefore either $\tau_{o}$ and $\tau_{i}$ are contained in $B_{1}$ or they remeet for the first time after $\tau_{o}$ leaves $B_{1}$ through $S_{i}$ and $\tau_{i}$ leaves $B_{1}$ through $S_{o}$. The latter case is impossible, since $\tau_{i}$ must cross $\tau_{o}$ before it can leave $B_{1}$ through $S_{i}$. It follows that the torus component is contained inside $B_{1}$.

The spherical caps $S_{i}$ in the double bubble and $S_{1}$ in the torus bubble are disjoint, since $S_{1}$ has boundary circle lying to the left of $S_{i}$, and each is convex in opposite directions. So no part of the torus bubble intersects the interior of the larger, rightmost component of the corresponding double bubble, which must then be contained in the ball component of the torus bubble. This proves the last assertion of the lemma.

COROLlary 4.10. In a minimizing equal-volume torus bubble, $\theta_{2}<120^{\circ}$.

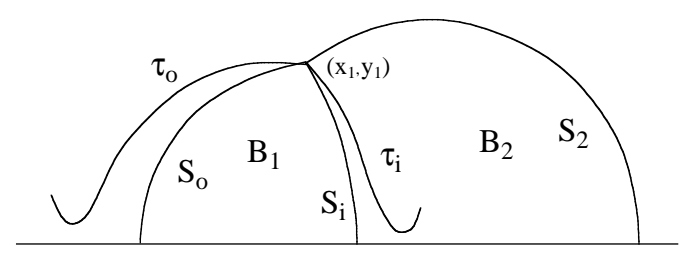

Figure 10. If $h_{o}$ is too small, a torus component cannot exist.

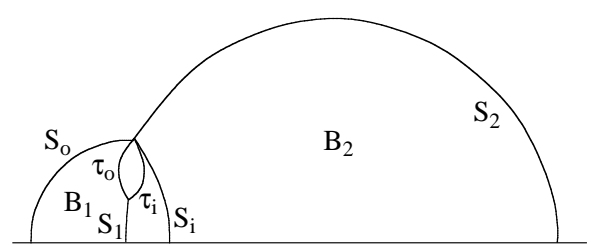

Figure 11. The torus component is trapped inside a component of an associated double bubble if $\theta_{2}>120^{\circ}$.

Proof. If $\theta_{2}>120^{\circ}$ then Lemma 4.9 implies that the volume of the torus component is strictly less than the volume of the smaller component of the 
double bubble whose spherical caps subtend angles $\theta_{2}$ and $\left(240-\theta_{2}\right)^{\circ}$. The other component of the torus bubble contains in its interior the larger component of this double bubble, which in turn contains more than half the total volume of the double bubble. It follows that the torus component has strictly less volume than the ball component of the torus bubble, contradicting the assumption of equal volume.

Recall that $\theta_{2}$ is the larger of the two angles subtended by the spherical caps, so the Corollary implies that both of $\theta_{1}$ and $\theta_{2}$ are less than $120^{\circ}$.

LEMMA 4.11. In a minimizing equal-volume torus bubble, $h_{o}>0$.

Proof. We know from Lemma 4.3 that $h_{o} \geq 0$. If $h_{o}=0$ then $\tau_{o}$ is a catenary. Since $y_{2} \geq y_{1}$ the catenary is given by a strictly increasing graph for $\left\{x \geq x_{2}\right\}$, implying in particular that $\theta_{2}>120^{\circ}$ which violates Lemma 4.9.

Knowledge of $h_{o}, \theta_{1}, \theta_{2}$ can be used to give a fairly accurate qualitative picture of the torus bubble.

Proposition 4.12. A minimizing equal-volume torus bubble has the following properties:

1. $\tau_{i}$ has negative slope at $\left(x_{1}, y_{1}\right)$ if and only if $\theta_{1}>30^{\circ}$. Its angle with the positive $x$-axis is $30^{\circ}-\theta_{1}$.

2. $\tau_{i}$ has positive slope at $\left(x_{2}, y_{2}\right)$ if and only if $\theta_{2}>30^{\circ}$. Its angle with the positive $x$-axis is $\theta_{2}-30^{\circ}$.

3. $\tau_{o}$ has positive slope at $\left(x_{1}, y_{1}\right)$ if and only if $\theta_{1}>60^{\circ}$. Its angle with the positive $x$-axis is $150^{\circ}-\theta_{1}$.

4. $\tau_{o}$ has angle at $\left(x_{2}, y_{2}\right)$ with the positive $x$-axis equal to $\theta_{2}-150^{\circ}$.

5. $\tau_{i}$ is a graph. It has a unique local minimum if and only if $\theta_{1} \geq 30^{\circ}$. It never has a local maximum. If $T_{i}$ is a nodoid then $h_{i}<0$.

6. $\tau_{o}$ always has a unique local maximum. It has a vertical tangent on the left if and only if $\theta_{1} \leq 60^{\circ}$. It never has a vertical tangent on the right. It never has a local minimum.

7. If $\theta_{1} \geq 30^{\circ}$ the local minimum $y_{\min }$ of $\tau_{i}$ has value

$$
y_{\min }=\frac{-f_{i}}{1+\sqrt{1+f_{i} h_{i}}},
$$

where $f_{i}$ is the force associated to $T_{i}$. 
8. The local maximum $y_{\max }$ of $\tau_{o}$ has value

$$
y_{\max }=\frac{1+\sqrt{1+f_{o} h_{o}}}{h_{o}},
$$

where $f_{o}$ is the force associated to $T_{o}$.

Proof. The first four assertions follow from the fact that the surfaces forming a torus bubble meet at $120^{\circ}$ angles along a triple curve.

If $\tau_{i}$ is not a graph then $\theta_{2}>120^{\circ}$, violating Corollary 4.10. If $\theta_{1} \geq 30^{\circ}$ then $\tau_{i}$ has nonpositive slope at $\left(x_{1}, y_{1}\right)$. Since $\theta_{2}>\theta_{1}$ the curve must have a local minimum in this case.

Suppose $\tau_{i}$ has a local maximum. Since $\theta_{2}>60^{\circ}, \tau_{i}$ has positive slope at $\left(x_{2}, y_{2}\right)$ and must have a local minimum as well. However $\theta_{2}>\theta_{1}$ so $\tau_{i}$ must be longer than a period, contradicting Proposition 4.7. So it has no local maximum.

If $h_{i} \geq 0$ and $T_{i}$ is a nodoid, then we can compare $T_{i}$ and $T_{o}$ to a double bubble whose spherical interface $S_{i}$ coincides with $S_{1}$, and apply Lemma 3.2 as in Lemma 4.9. $T_{i}$ is trapped inside the component $B_{2}$ to the right of the interface and $T_{o}$ is trapped inside the component $B_{1}$ to the left of the interface, as in Figure 12. In particular, $h_{o}<0$ in this case, a contradiction. So if $T_{i}$ is a nodoid then $h_{i}<0$.

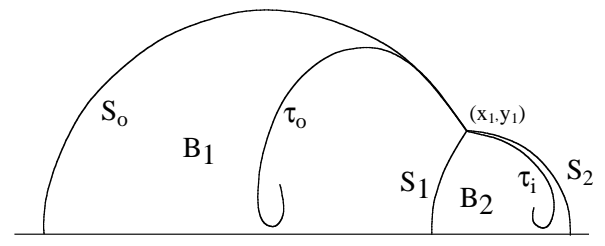

Figure 12. A double bubble traps $T_{i}$ and $T_{o}$ if $T_{i}$ is a nodoid with $h_{i}>0$.

If $\theta_{1}<30^{\circ}$ then $\tau_{i}$ has positive slope at $\left(x_{1}, y_{1}\right)$. If it has a minimum then it must pass through a maximum first, so it has no minimum.

$\tau_{o}$ is always oriented upwards at $\left(x_{1}, y_{1}\right)$ and downwards at $\left(x_{2}, y_{2}\right)$, so it always has a local maximum. If it has a local minimum then it would contain two local maxima, violating Proposition 4.7. If $\theta_{1}<60^{\circ}$ then the curve starts out to the left, therefore it has a vertical tangent at which it changes direction from left to right. Proposition 4.6 implies that there cannot be a second vertical tangent, so it has no vertical tangent on the right, as claimed. If $\theta_{1} \geq 60^{\circ}$ then $\tau_{o}$ initially is going right. If it has a vertical tangent on the right then the angle $\theta_{2}$ must be less than $60^{\circ}$, contradicting the assumption that $\theta_{1} \leq \theta_{2}$. This proves the assertion concerning vertical tangents of $\tau_{o}$. 
To calculate the local minimum $y_{\min }$ of $\tau_{i}$, we use equation 2 of Section 3 for the force of $T_{i}$,

$$
f_{i}=h_{i} y^{2}-2 y \cos \alpha .
$$

At a minimum, $\alpha=0$ and $\cos \alpha=1$, so

$$
f_{i}=h_{i} y^{2}-2 y \text {. }
$$

If $h \neq 0$ this quadratic expression gives two roots for $y$ :

$$
y=\frac{1 \pm \sqrt{1+h_{i} f_{i}}}{h_{i}} .
$$

The value of the minimum is given by

$$
\frac{1-\sqrt{1+h_{i} f_{i}}}{h_{i}}
$$

whatever the sign of $h_{i}$. If $h_{i} f_{i}>0$, as Corollary 3.3 implies happens at a local minimum for a nodoid, then the other root is negative and meaningless. If $h_{i} f_{i}<0$, as Corollary 3.3 implies happens at a local minimum for an unduloid, then the other root corresponds to the local maximum of the unduloid. A double root occurs only in the case of a cylinder. equation 12 becomes linear when $h_{i}=0$. An equivalent expression for the minimum, which holds also when $h_{i}=0$, is

$$
y_{\min }=\frac{-f_{i}}{1+\sqrt{1+f_{i} h_{i}}} .
$$

For a local maximum, a similar analysis gives that $y_{\max }$ is one of the two roots

$$
y_{\max }=\frac{1 \pm \sqrt{1+f_{o} h_{o}}}{h_{o}} .
$$

Since by Corollary $4.19 T_{o}$ is always a nodoid, $h_{o}>0$ and $f_{o}>0$, there is a unique positive root, and

$$
y_{\max }=\frac{1+\sqrt{1+f_{o} h_{o}}}{h_{o}} .
$$

Proposition 4.13. Given $\theta_{1}$ and $h_{o}$, there is at most one corresponding minimizing equal-volume torus bubble.

Proof. Given $\theta_{1}$ and $h_{o}, h_{i}=2-h_{i}$ is also determined. Thus $S_{1}, T_{o}$ and $T_{i}$ are uniquely determined. The spherical cap $S_{2}$ is uniquely determined by the second intersection point $\left(x_{2}, y_{2}\right)$ of $\tau_{o}$ and $\tau_{i}$, assuming that this second point exists. If $\tau_{o}$ and $\tau_{i}$ do not meet in a second point then there is no torus bubble corresponding to $\theta_{1}$ and $h_{o}$. In general, $\tau_{o}$ and $\tau_{i}$ may intersect in many points, and it is necessary to show that only one of these can possibly lead to a torus bubble. 
If $\tau_{o}$ and $\tau_{i}$ are graphs, then it is clear that their first intersection point with $x$ coordinate larger than $x_{1}$ is the unique intersection point which can give a torus bubble. In general, $\tau_{i}$ is a graph, but not $\tau_{o}$. However Proposition 4.12 shows that $\tau_{o}$ has no vertical tangents for $x>x_{1}$, so that both curves are graphs in this region and the same argument applies.
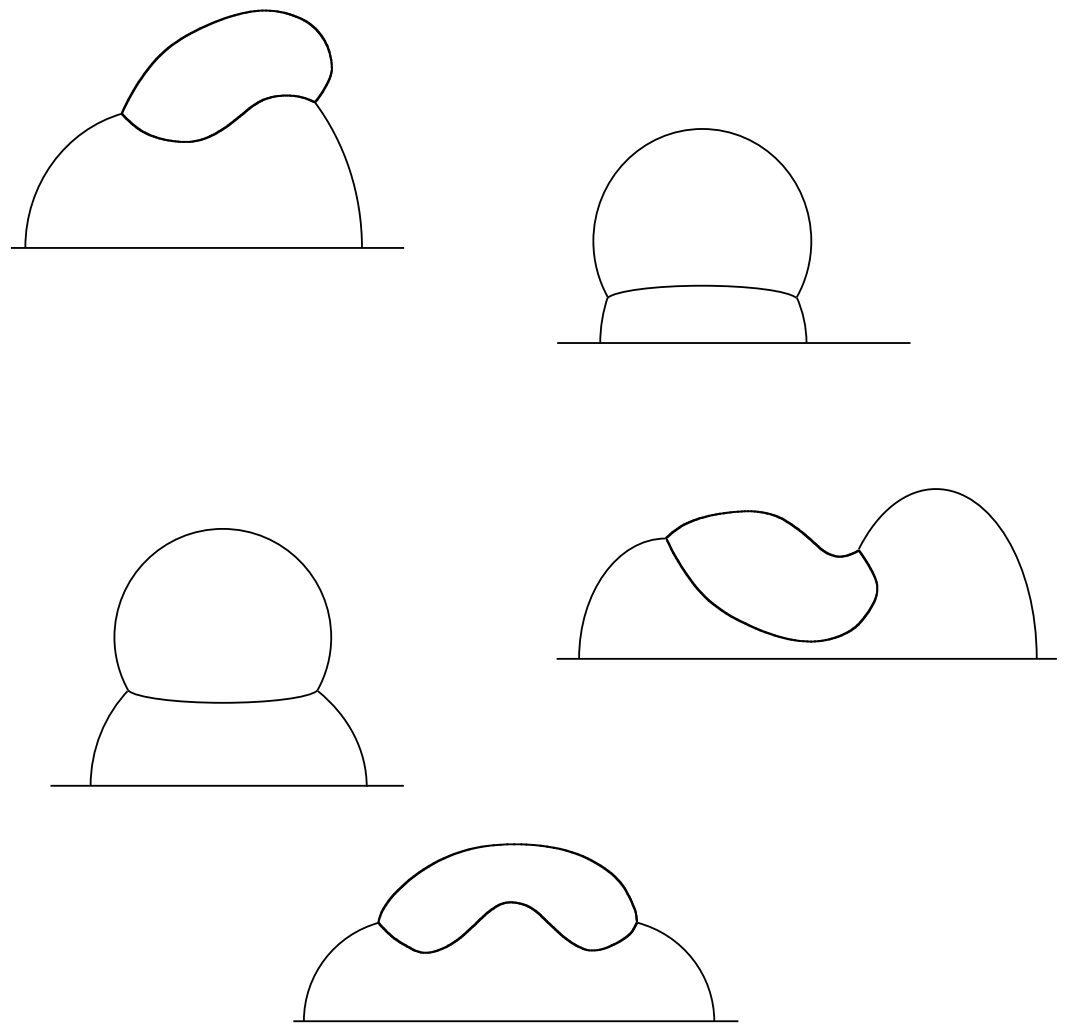

Figure 13. Some eliminated possibilities for cross-sections of torus bubbles.

Proposition 4.14. If a torus bubble has $\theta_{1}+\theta_{2}>180^{\circ}$, then it is not a minimizer.

Proof. Under this assumption there is a value of $y$ realized by three distinct points on the circular arcs generating the spherical caps. Since $\theta_{2} \geq \theta_{1}$ we have in particular that $\theta_{2}>90^{\circ}$. We know from Lemma 4.9 that $\theta_{2}<120^{\circ}$. So $90^{\circ}<\theta_{2}<120^{\circ}, 60^{\circ}<\theta_{1} \leq 120^{\circ}$. Then Lemma 4.12 implies that the curves $\tau_{i}$ and $\tau_{o}$ are both graphs. If $\theta_{1}+\theta_{2}>180^{\circ}$ then there are three points on $S_{1} \cup S_{2}$ with $y$-value equal to $y_{2},\left(a_{1}, y_{2}\right),\left(a_{2}, y_{2}\right),\left(a_{3}, y_{2}\right)$, with $a_{1}<a_{2}<a_{3}$ and $a_{2}=x_{2}$. Reflect the part of the bubble between the planes $x=a_{1}$ and 
$x=a_{2}$ through the plane $x=\left(x_{1}+x_{2}\right) / 2$ to get a new piecewise-smooth surface with the same area enclosing the same volumes, as in the proof of Lemma 4.7, but without needing to worry here about introducing self-intersections. The new piecewise-smooth surface has three surfaces meeting at an angle not equal to $120^{\circ}$ and so is not minimizing, a contradiction.

Propositions 4.6, 4.7, 4.14, 4.12, Lemma 4.8 and Corollary 4.10 combine to rule out many possibilities for the shapes of minimizing torus bubbles. See Figure 13 for cross-sections of some of these eliminated possibilities.

LEMMA 4.15. For any constant $b$, the function $f(c)=b\left(1-c^{2}\right)+$ $c \sqrt{3\left(1-c^{2}\right)}$ is at most two-to-one on $(-1,1)$.

Proof. $f^{\prime}(c)=-2 b c-\sqrt{3} g(c)$, where

$$
g(c)=\left(2 c^{2}-1\right) / \sqrt{1-c^{2}}
$$

Since $g^{\prime \prime}(c)=3\left(1-c^{2}\right)^{-5 / 2}>0, g$ is convex on $(-1,1)$.

A line intersects a convex curve at most twice, so $f^{\prime}$ has at most two roots on $(-1,1)$. Thus $f$ has at most one local maximum and one local minimum on $(-1,1)$. Combined with $f(0)=f(1)=0$, this completes the proof.

We calculate in the next proposition the value of the forces $f_{o}$ of $T_{o}$ and $f_{i}$ of $T_{i}$.

Proposition 4.16. For the torus bubble determined by $\theta_{1}$ and $h_{o}$, the forces $f_{o}$ of $T_{o}$ and $f_{i}$ of $T_{i}$ are given by:

$$
f_{o}=\left(h_{o}-1\right)\left(1-c_{1}^{2}\right)+c_{1} \sqrt{3\left(1-c_{1}^{2}\right)}
$$

and

$$
f_{i}=\left(h_{i}-1\right)\left(1-c_{1}^{2}\right)-c_{1} \sqrt{3\left(1-c_{1}^{2}\right)}=-f_{o}
$$

where $c_{1}=\cos \theta_{1}$.

Proof. We first calculate the force $f_{o}$ of $T_{o}$. At $\left(x_{1}, y_{1}\right), y_{1}=\sin \theta_{1}$ and $\alpha=5 \pi / 6-\theta_{1}$ is the angle between the positive $x$-axis and the graph of $\tau_{o}$, therefore

$$
\begin{aligned}
f_{o} & =h_{o} y_{1}^{2}-2 y_{1} \cos \alpha \\
& =h_{o} \sin ^{2} \theta_{1}-2 \sin \theta_{1} \cos \left(5 \pi / 6-\theta_{1}\right) \\
& =h_{o}\left(1-\cos ^{2} \theta_{1}\right)-2 \sin \theta_{1}\left((-\sqrt{3} / 2) \cos \theta_{1}-(1 / 2) \sin \left(-\theta_{1}\right)\right) \\
& =h_{o}\left(1-c_{1}^{2}\right)+c_{1} \sqrt{3\left(1-c_{1}^{2}\right)}-\left(1-c_{1}^{2}\right) \\
& =\left(h_{o}-1\right)\left(1-c_{1}^{2}\right)+c_{1} \sqrt{3\left(1-c_{1}^{2}\right)}
\end{aligned}
$$


Similarly,

$$
\begin{aligned}
f_{i} & =h_{i} y_{1}^{2}-2 y_{1} \cos \alpha \\
& =\left(h_{i}-1\right)\left(1-c_{1}^{2}\right)-c_{1} \sqrt{3\left(1-c_{1}^{2}\right)} .
\end{aligned}
$$

Since $h_{o}-1=1-h_{i}=-\left(h_{i}-1\right)$ by Lemma $4.3, f_{o}=-f_{i}$.

Note. The fact that $f_{o}=-f_{i}$ is an example of a general "balancing principle" for constant mean curvature surfaces; see [25].

Lemma 4.17. For the torus bubble determined by $\theta_{1}$ and $h_{o}$, the angle $\theta_{2}$ is one of the (at most) two solutions of the equation

$$
f\left(\cos \theta_{1}\right)=f\left(\cos \theta_{2}\right),
$$

where

$$
f(c)=\left(h_{o}-1\right)\left(1-c^{2}\right)+c \sqrt{3\left(1-c^{2}\right)} .
$$

Proof. We calculate the force $f_{o}$ of $T_{o}$ at both $\left(x_{1}, y_{1}\right)$ and $\left(x_{2}, y_{2}\right)$.

At $\left(x_{1}, y_{1}\right)$,

$$
f_{o}=\left(h_{o}-1\right)\left(1-c_{1}^{2}\right)+c_{1} \sqrt{3\left(1-c_{1}^{2}\right)},
$$

where $y_{1}=\sin \theta_{1}$ and $c_{1}=\cos \theta_{1}$. A similar calculation at $\theta_{2}$ shows

$$
f_{o}=\left(h_{o}-1\right)\left(1-c_{2}^{2}\right)+c_{1} \sqrt{3\left(1-c_{2}^{2}\right)} .
$$

Setting the two forces equal and applying Lemma 4.15 gives that there are at most two possible values for $c_{2}=\cos \theta_{2}$. Since $0<\theta_{2}<180^{\circ}$, where the cosine function is monotonically decreasing, this implies that there are at most two possible values for $\theta_{2}$.

One solution occurs when the angles are equal. An example of such a symmetric torus bubble, depicted in Figure 7, occurs with $\theta_{1}=\theta_{2}=90^{\circ}$, and $h_{o}$ approximately 1.9848. However these symmetric solutions never lead to a minimizing torus bubble by Corollary 4.5.

Recall that we remarked after Corollary 4.5 that we can without loss of generality restrict attention to nonsymmetric torus bubbles. This means that we are working in the case where $\theta_{1}<\theta_{2}$. For each $\theta_{1}, h_{o}$, there is at most one value of $\theta_{2}$ which can potentially lead to a stable torus bubble.

Proposition 4.18. If a minimizing torus bubble has $60^{\circ} \leq \theta_{1} \leq 120^{\circ}$ then $h_{o}>1-\sqrt{3} \cot \theta_{1}$ and $h_{i}<1+\sqrt{3} \cot \theta_{1}$. The same statement holds with $\theta_{2}$ replacing $\theta_{1}$. 
Proof. We again apply Lemma 3.2 to compare the torus bubble with a double bubble whose smaller spherical cap $S_{o}$ subtends an angle of $\theta_{1}$, as in the proof 1 Lemma 4.9. See Figure 14. If $h_{i}$ is larger than the mean curvature of $S_{i}$, then $T_{i}$ is a nodoid contained inside the smaller component of the double bubble, as shown in Lemma 3.2. See Figure 14. It follows that $\tau_{o}$ cannot remeet $\tau_{i}$ and the two curves cannot generate a torus component.

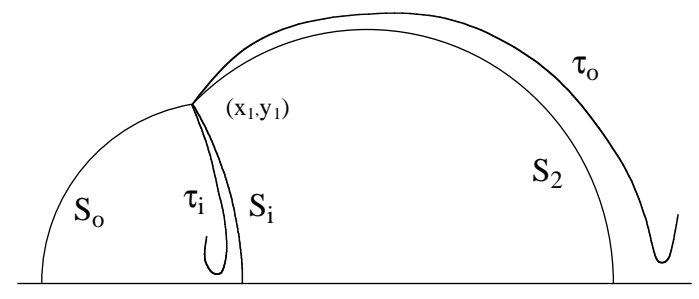

Figure 14. The mean curvature of $T_{o}$ cannot be too small if $\tau_{o}$ and $\tau_{i}$ are to meet again.

Corollary 4.19. $T_{o}$ is a nodoid.

Proof. If $60^{\circ} \leq \theta_{1} \leq 120^{\circ}$ then Proposition 4.18 shows that the mean curvature of $T_{o}$ is greater than that of the sphere which is tangent to it at $\left(x_{1}, y_{1}\right)$, so Lemma 3.2 implies that $T_{o}$ is a nodoid. If $\theta_{1}<60^{\circ}$ then $\tau_{o}$ starts out heading to the left. It must become vertical, hence $T_{o}$ must be a nodoid.

LEMMA 4.20. Let $N$ be a subcurve of a nodary generating a nodoid of mean curvature $h>0$, running between a vertical tangency on the left at $\left(x_{v}, y_{v}\right)$ and a vertical tangency on the right at $\left(x_{v}^{\prime}, y_{v}^{\prime}\right)$, with a unique local maximum at $\left(x_{M}, y_{M}\right)$. Then a circle $C$ of curvature $h$ which is tangent to $N$ at $\left(x_{v}, y_{v}\right)$ lies underneath $N$. Moreover $x_{v}-x_{m}$ is greater than the radius $1 / h$ of the circle.

Proof. In a deleted neighborhood of $\left(x_{v}, y_{v}\right)$, the curvature of $N$ is less than that of $C$, so that $C$ locally lies beneath $N$. If $C$ crosses $N$ between $x_{v}$ and $x_{v}^{\prime}$, let $C^{\prime}$ be the subcurve of $C$ starting at $\left(x_{v}, y_{v}\right)$ and running to the first point of intersection of $C$ with $N$. Let $C_{t}^{\prime}=C^{\prime}+(0, t)$ be a vertical translate of $C^{\prime}$ by a distance $t$ along the $y$-axis. Let $T=\sup \left\{t: C_{t}^{\prime} \cap N \neq \emptyset\right\}$. Thus $C_{T}^{\prime}$ is tangent to $N$ at a point $P$ and lies above $N$ near $P$. The curvature of $N$ at $P$ is given by $k_{m}=h-k_{p}<h$. Since the curvature of $C_{T}^{\prime}$ is greater than that of $N$, it cannot lie above it, and it follows that $x_{M}-x_{v}$ is larger than the radius of $C$, i.e., $x_{M}-x_{v}>1 / h$. 
The next proposition rules out the existence of minimizing torus bubbles with $h_{o}$ and $\theta_{1}$ both close to 0 . The numerical calculations that we will apply are badly behaved in this region, so we use this geometric argument to exclude it.

Proposition 4.21. If a torus bubble has $h_{o} \leq 0.2$ and $\theta_{1} \leq 5.7^{\circ}$, then it is not a minimizer.

Proof. We will show that before $\tau_{i}$ and $\tau_{o}$ rejoin at $\left(x_{2}, y_{2}\right)$, either $\tau_{o}$ has two vertical tangencies, or $\tau_{i}$ traverses more than a full period. Note that our assumption implies that $h_{i} \geq 1.8$

Proposition 3.4 implies that $\tau_{i}$ is a graph and its period is no longer than $2 \pi / h_{i}<3.5$. The bubble is not a minimizer if $x_{2}-x_{1}>3.5$ by Proposition 4.7. The initial angle of $\tau_{i}$ from the positive $x$-axis is $30^{\circ}-\theta_{1}$, so it cannot turn (anti-clockwise) through an angle of more than $60^{\circ}+\theta_{1}$. When they meet again $\tau_{i}$ and $\tau_{o}$ intersect at an angle of $120^{\circ}$. It follows that $\tau_{o}$ does not intersect $\tau_{i}$ before it turns (clockwise) through an angle greater than $180^{\circ}-\theta_{1}$. Therefore the two curves cannot intersect before the nodary $\tau_{o}$ reaches its maximum, which happens when $\tau_{o}$ has turned through an angle of $150^{\circ}-\theta_{1}$. Let $\left(x_{M}, y_{M}\right)$ be the point where $\tau_{o}$ reaches its maximum and $\left(x_{v}, y_{v}\right)$ the point where $\tau_{o}$ first goes vertical. Then $x_{2}-x_{1}>x_{M}-x_{1}$.

Now $h_{o}=k_{p}+k_{m}$ where $k_{m}$ is the curvature of the generating curve in the $x y$ plane and $k_{p}$ is the principal curvature due to rotation around the axis. At $\left(x_{v}, y_{v}\right), k_{p}=0$ and $h_{o}=k_{m} \leq .2$ by hypothesis.

Lemma 4.20 implies that $x_{M}-x_{v}>1 / h_{o} \geq 5$.

The assumptions $h_{o} \leq .2$ and $\theta_{1} \leq 5.7^{\circ}$ give an upper bound for the force $f_{o}$ of $T_{o}$. Calculating at height $y_{1}=\sin \theta_{1}<\theta_{1} \leq 5.7 \pi / 180<.1$ gives

$$
\begin{aligned}
\left|f_{o}\right| & =\left|y_{1}{ }^{2} h_{o}-y_{1} \cos \left(30^{\circ}+\theta_{1}\right)\right| \\
& <\left|y_{1}{ }^{2} h_{o}-y_{1} \sqrt{3} / 2\right| \\
& <(.1)^{2} h_{o}+.1 \sqrt{3} / 2 \\
& <.09
\end{aligned}
$$

We bound the value of $y_{v}$ from above by solving $f_{o}=y_{v}^{2} h_{o}$.

$$
y_{v}^{2}=f_{o} / h_{o}<.09 / h_{o},
$$

so

$$
y_{v}<\sqrt{.09 / h_{o}}
$$

We next bound $x_{1}-x_{v}$ from above by noting that $\tau_{o}$ is convex between $x_{v}$ and $x_{1}$ by Lemma 3.4. This implies that $\left(x_{v}, y_{v}\right)$ lies to the right of the point 
$\left(x, y_{v}\right)$ at which the line of slope $\dot{\tau}_{o}\left(x_{1}\right)$ has height $y_{v}$, so that

$$
\begin{aligned}
\left|x_{v}-x_{1}\right|<\left|x-x_{1}\right| & =\left|\left(y_{v}-y_{1}\right) / \dot{\tau}_{o}\left(x_{1}\right)\right|<\sqrt{3}\left(y_{v}-y_{1}\right) \\
& <\sqrt{3} y_{v}<\sqrt{3} \sqrt{.09 / h_{o}}=\sqrt{.27 / h_{o}} .
\end{aligned}
$$

This implies that

$$
x_{M}-x_{1}=\left(x_{M}-x_{v}\right)-\left(x_{1}-x_{v}\right)>1 / h_{o}-\sqrt{.27 / h_{o}} .
$$

For $0<h_{o} \leq .2$, differentiation shows that the function $1 / h_{o}-\sqrt{.27 / h_{o}}$ is decreasing and its minimum value occurs at $h_{o}=.2$, so that $x_{M}-x_{1}>$ $1 /(0.2)-\sqrt{.27 /(0.2)}>3.8$. Thus $\tau_{o}$ travels a distance of at least 3.8 to the right before hitting $\tau_{i}$. But the period of $\tau_{i}$ is less than 3.5. We have shown that either $\tau_{o}$ changes direction and has two vertical tangencies or $\tau_{i}$ contains a full period. In the first case it is not a minimizer by Lemma 4.8, and in the second case it is not a minimizer by Proposition 4.7.

The next lemma will be used to give bounds on the size of a circle enclosing a loop of a nodary.

LEMMA 4.22. If an embedded curve $\gamma$ in the positive quadrant of the plane is tangent to the $y$-axis at the origin and has curvature $k>1 / r>0$, then it does not meet $C(r)$, the circle of radius $r$ centered at $(0, r)$, at any point other than the origin.

Proof. If it does cross $C(r)$, denote by $\gamma^{\prime}$ the subcurve of $\gamma$ starting at the origin and running to the first point of intersection with $C(r)$, as in Figure 15. The subcurve $\gamma^{\prime}$ is a graph over the $x$-axis since it is embedded. Let $\gamma_{t}^{\prime}=\gamma^{\prime}+(0, t)$ be a vertical translate of $\gamma^{\prime}$ by a vertical distance of $t$. Let $T=\sup \left\{t: \gamma_{t}^{\prime} \cap C(r) \neq \emptyset\right\}$. Then $\gamma_{T}^{\prime}$ meets $C(r)$ at a point $P$ in its interior, but not the interior of $C(r)$, and thus is tangent to $C(r)$ at $P$. Since the curvature of $\gamma_{T}^{\prime}$ is greater than that of $C(r)$, this is a contradiction.
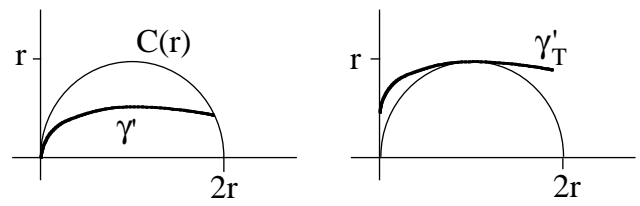

Figure 15. The curvature of the curve $\gamma$ cannot be greater than $1 / r$ if it crosses $C(r)$ twice.

LEMMA 4.23. If $\tau_{o}$ has curvature greater than $k$, and $\tau_{i}$ has curvature less than $-k$, then their union is contained in the intersection of two circles of radius $r=1 / k$ meeting at $120^{\circ}$. 
Proof. Construct circles $C_{1}$ and $C_{2}$ of radius $1 / k$, meeting at $120^{\circ}$ at $\left(x_{1}, y_{1}\right)$, with $C_{1}$ tangent to $\tau_{o}$ and $C_{2}$ tangent to $\tau_{i}$ as in Figure 16 . Let $D$ denote the line segment joining the two intersection points of $C_{1}$ and $C_{2}$. By Lemma 4.22 we know that each of $\tau_{o}$ and $\tau_{i}$ cannot leave the intersection of these two circles without first crossing $D$. Since each is convex in $C_{1} \cap C_{2}$ it follows that they remeet before either one leaves $C_{1} \cap C_{2}$. It follows that the intersection of the torus component with the upper half-plane is contained in $C_{1} \cap C_{2}$.

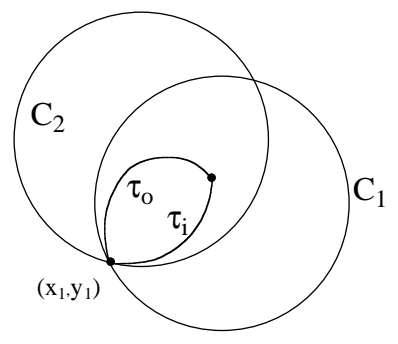

Figure 16. The torus component generating curves $\tau_{i}$ and $\tau_{o}$ are trapped inside two circles meeting at $120^{\circ}$.

LEMMA 4.24. If a torus bubble has $h_{i} \leq-k<0$ and the intersection of the torus component with the upper half-plane has minimum y-value equal to $y_{0}$ with $y_{0}>1 / k$, then the torus component contains volume

$$
v \leq 2.5 \pi\left(y_{0}+\frac{\sqrt{3}}{2} \frac{y_{0}}{k y_{0}-1}\right)\left(\frac{y_{0}}{k y_{0}-1}\right)^{2} .
$$

Proof. In a torus bubble with $h_{i} \leq-k<0$ we have that $\left|h_{o}\right|=-h_{i}+$ $2 \geq k$. For a Delaunay curve passing through a point $(x, y)$ in the upper half-plane,

$$
\begin{aligned}
\left|k_{m}\right| & \geq|h|-\left|k_{p}\right| \\
& =|h|-\frac{1}{y \sqrt{1+\dot{y}^{2}}} \\
& \geq|h|-\frac{1}{y} .
\end{aligned}
$$

For the curves $\tau_{i}$ and $\tau_{o}$ we have $y \geq y_{0}$, so that

$$
\left|k_{m}\right| \geq k-\frac{1}{y_{0}} .
$$

So the curves $\tau_{o}$ and $\tau_{i}$ each have curvature with absolute value greater than $\left(k y_{0}-1\right) / y_{0}$. 
By Lemma 4.23 the intersection of the torus component with the upper half-plane is contained in the intersection $w$ of two circles of radius $r=$ $y_{0} /\left(k y_{0}-1\right)$ meeting at an angle of $120^{\circ}$. The area of $w$ is

$$
a=(2 \pi / 3-\sqrt{3} / 2) r^{2}<1.25\left(\frac{y_{0}}{k y_{0}-1}\right)^{2} .
$$

The diameter of $w$ is

$$
d=\sqrt{3} \frac{y_{0}}{k y_{0}-1} .
$$

Since $w$ is centrally symmetric and has minimal $y$-value smaller than or equal to $y_{0}$, the center of mass of $w$ has $y$-coordinate at most $y_{0}+d / 2$. Pappus' theorem for the volume of a solid of revolution obtained by rotating this area around the $x$-axis gives that

$$
\begin{aligned}
v & \leq 2 \pi\left(y_{0}+\frac{d}{2}\right) 1.25\left(\frac{y_{0}}{k y_{0}-1}\right)^{2}, \\
& \leq 2.5 \pi\left(y_{0}+\frac{\sqrt{3}}{2} \frac{y_{0}}{k y_{0}-1}\right)\left(\frac{y_{0}}{k y_{0}-1}\right)^{2} .
\end{aligned}
$$

We apply these volume estimates to reduce the range of possible mean curvatures in a minimizing equal-volume torus bubble.

Proposition 4.25. In a minimizing equal-volume torus bubble, $h_{o} \leq 10$.

Proof. The hypothesis $h_{o} \leq 10$ is equivalent to $h_{i} \geq-8$. Suppose to the contrary that $h_{i}<-8$ in a minimizing equal-volume torus bubble.

Proposition 4.12 states that $\tau_{i}$ is a graph, so that the volume $v_{i}$ of the ball component $B$ is strictly larger than the volume of the region under $S_{2}$. Lemmas 4.9 and 4.8 imply that $60^{\circ}<\theta_{2}<120^{\circ}$. It follows that $v_{i}>5 \pi / 24$, the volume under a unit radius spherical cap subtending an angle of $60^{\circ}$. We now estimate the volume $v_{o}$ of the torus component. The curves $\tau_{i}$ and $\tau_{o}$ generating the torus component contain the point $\left(x_{2}, y_{2}\right)$ where $\sqrt{3} / 2 \leq y_{2} \leq$ 1. Their maximum and minimum $y$-values differ by less than $2 /\left|h_{i}\right| \leq 0.25$ by Proposition 3.4, and therefore both curves are contained in $\{y \geq \sqrt{3} / 2-0.25\}$. The expression $\frac{y_{0}}{k y_{0}-1}$ is decreasing with respect to $y_{0}$ when $y_{0}>1 / k$, and decreasing with respect to $k$, and also $0.6<\sqrt{3} / 2-0.25$. We apply Lemma 4.24 with $k \geq 8$ and $y_{0} \geq 0.6$, giving that

$$
\begin{aligned}
v_{o} & <2.5 \pi\left(y_{0}+\frac{\sqrt{3}}{2} \frac{y_{0}}{k y_{0}-1}\right)\left(\frac{y_{0}}{k y_{0}-1}\right)^{2} \\
& <2.5 \pi\left(1+\frac{\sqrt{3}}{2} \frac{0.6}{4.8-1}\right)\left(\frac{0.6}{4.8-1}\right)^{2} \\
& <0.08 \pi .
\end{aligned}
$$


Thus $v_{o}<0.08 \pi<5 \pi / 24<v_{i}$, contradicting the assumption that the torus bubble encloses equal volumes.

Our algorithm will need the following rather technical result about the slope of a generating curve.

Lemma 4.26. Suppose that $\tau_{i}$ satisfies $h_{i} \leq 2$ and $0 \leq \theta_{1} \leq 4^{\circ}$. Let $\alpha$ be the angle made by $\tau_{i}$ with the positive $x$-axis and let $\alpha_{1}$ be the value of this angle at $\left(x_{1}, y_{1}\right)$. Then $\alpha \geq \alpha_{1}$ for as long as $\tau_{i}$ remains below height $\{y=1 / 2\}$. In particular, $\alpha \geq 26^{\circ}$ below this height.

Proof. First note that if $h_{i} \leq 0$ then $\tau_{i}$ is a nodary or catenary, and the angle made by $\tau_{i}$ with the positive $x$-axis is increasing as one moves along $\tau_{i}$, which implies the conclusion of the lemma. So we can assume that $0<h_{i} \leq 2$.

Let $y$ be any point on $\tau_{i}$. Then

$$
h_{i} y^{2}-2 y \cos \alpha-f_{i}=0 .
$$

A given value of $\cos \alpha$ is realized by at most two $y$ values solving this quadratic equation. Suppose that as the $\tau_{i}$ goes from a minimum to a maximum, the angle $26^{\circ} \leq \alpha_{1}=30^{\circ}-\theta_{1} \leq 30^{\circ}$ occurs twice. The two $y$-values which share the same $\alpha_{1}$ both solve the above equation. Thus they sum to $2 \cos \alpha_{1} / h_{i} \geq$ $2(.8) / 2 \geq .8$. Since the smaller root has $y$-coordinate $\sin \left(4^{\circ}\right)<.1$ the larger root is greater than $.7>1 / 2$. The angle $\alpha$ is increasing near the smaller root. Thus $\tau_{i}$ subtends an angle of at least $\alpha_{1}$ with the $x$-axis as long as $\tau_{i}$ remains below height $1 / 2$. Since $\alpha_{1}=30^{\circ}-\theta_{1} \geq 26^{\circ}$, the final conclusion holds.

\section{Computation}

We have shown that to solve Conjecture 3 in the equal volume case it suffices to show that certain torus bubbles are less efficient at enclosing two equal volumes then a double bubble. In this section we will show how these torus bubbles are ruled out by a computation. The algorithm for this computation is given below.

The basic idea is to consider a domain of torus bubbles corresponding to a product of small intervals in each of $\theta_{1}$ and $h_{o}$, and to calculate as much as possible about the geometry of the corresponding torus bubbles. Various calculations are then applied which rule these torus bubbles out as potential minimizers. The accuracy of these calculations depends on the size of the domain rectangle we start with. The computational scheme will succeed if these can be chosen small enough to get sufficient accuracy, yet large enough that a reasonable number of them cover all the possibilities.

The computation was performed using double precision floating point numbers. The fundamental data type used is the IEEE 754 32-bit real number, 
see [5]. This is a binary representation with a 23-bit mantissa (plus an implied leading bit), an 8-bit binary exponent, and a sign bit. The (finitely many) real numbers that can be represented in this standard with no error are called representable. The IEEE standard specifies that the add, subtract, multiply, divide, and square root operations be performed as if done exactly and rounded to the nearby representable number according to the rounding mode in effect. There are three rounding modes that can be chosen: up, down, or nearest. The values $+\infty$ and $-\infty$ are representable and behave in a specified way. Floating point exceptions are masked, but flags are sticky and available for clearing and inspection. Most computers in use today implement the IEEE standard. We denote the result of rounding a real number $x$ down to a representable number smaller or equal to $x$ by $\underline{x}$, and similarly denote by $\bar{x}$ the result of rounding $x$ up to a representable number greater or equal to $x$.

Combined with the methods of interval arithmetic, see Moore [29] and Alefeld-Herzberger [2], the IEEE standard allows numerical calculation with exact bounds on accuracy. Interval arithmetic is a method by which a realvalued function on the reals can be extended to an interval-valued function of intervals. An interval is formed from two representable reals. Mathematically, it represents the closed interval between the two reals. The add, subtract, multiply, divide, and square root functions are extended to intervals by the IEEE operations on reals along with directed rounding. We only use the round-tonearest mode in calculating averages, (see the procedure avgwt below), as these are only used to divide intervals and do not need to have bounds calculated.

Generally, we say that an interval $X$ is an extension of a real number $x$ if $x \in X$ and an interval-valued function of intervals $F$ is an extension of $f$ if $x \in X \Rightarrow f(x) \in F(X)$.

In this section, real numbers are denoted by lower case, and intervals with representable real endpoints by upper case. The reals embed into the set of intervals by mapping a real to the smallest interval containing it. The lower and upper bounds to an interval are denoted with lower and upper bars, so $X=[\underline{X}, \bar{X}]$. Arithmetic operations on representable reals and intervals are interpreted according to IEEE and interval rules, not by the usual mathematical definitions.

As an example, the sum of two intervals $A=[\underline{A}, \bar{A}]$ and $B=[\underline{B}, \bar{B}]$ is given by

$$
A+B=[\underline{\underline{A}}+\underline{B}, \overline{\bar{A}+\bar{B}}],
$$

where the left endpoints are rounded down when added, and the right endpoints are rounded up when added.

Other operations on reals are extended to intervals as in [29] and [2]. Relations are interpreted positively, so for example when $X$ and $Y$ are intervals, 
$X<Y$ means that for any $x \in X$ and any $y \in Y, x<y$, and $X \neq Y$ means that for any $x \in X$ and any $y \in Y, x \neq y$.

We have equivalent expressions $X<Y \Leftrightarrow \bar{X}<\underline{Y}$, and $X \neq Y \Leftrightarrow$ $X \cap Y=\emptyset$. The union of intervals $X$ and $Y$ is somewhat nonstandard; it is the smallest interval containing both sets, and is denoted $X \cup Y$. The intervalvalued functions Absolute $\operatorname{Value}(X), \operatorname{Max}(X, Y), \operatorname{Min}(X, Y)$, and the intersection of two intervals $X \cap Y$ are defined in the standard way, without rounding. There is no IEEE standard for transcendental functions, so we designed our program to avoid all calls to trigonometric functions.

The operations of the IEEE floating point standard can lead to undefined operations. For example the quotient $0 / 0$ and the product $0 \times+\infty$ result in an output of NAN (Not A Number), and are signaled by the presence of an exception flag. Operations of interval arithmetic can also lead to questionable operations, for example when the quotient of two intervals $A / B$ is calculated and $B$ contains 0 . Our implementation of the division operator on intervals returns $[-\infty,+\infty]$ if $B$ contains 0 . The algorithm draws no conclusions when such a division occurs, but rather calls for a subdivision of the input into intervals of smaller size, where the operations are repeated with greater accuracy. Exceptions such as overflow and underflow, sometimes an issue in computer assisted proofs, are not an issue in our algorithm. In any case they do not occur.

Bounds obtained from repeated applications of interval arithmetic are potentially far from sharp, especially when using wide input intervals. If just a single value needs to be computed rigorously, one can work with intervals whose width is comparable to the roundoff error of a single operation. For our purposes these types of intervals are far too thin, and would make the number of calculations we need impractical. Our intervals are "fat" in the terminology of Fefferman [15], meaning that they are sized by the scope of the problem we are solving rather than the size of the computational rounding. Often the intervals are wide enough to make a perfectly good formula look like nonsense. Sometimes it is possible to narrow the intervals under consideration because we have knowledge about what the possible legitimate values are that could arise during a computation. For example, consider a formula involving square roots. Assuming we're not using imaginary numbers, the validity of the formula presupposes that the argument to the square root is nonnegative. However, when we pass to an interval extension of the formula, the interval argument to the square root will often include negative values, even though these values cannot arise from the problem we are interested in. The square root of a negative number would normally trigger an exception, but the nature of the calculation allows us to define the interval square root function Sqrt to discard any negative portion of an interval argument. The justification for this apparent sleight-of-hand rests on the validity of the original formula over the 
reals. If our theorems tell us that the quantity whose square root we are taking is nonnegative, we are justified in truncating the interval to exclude negatives.

The interval function which always returns $[-\infty,+\infty]$ can be used to represent any function, but not very usefully. We use this interval to return the value of a division by an interval containing zero. An interval $[a, b]$ is empty if $a>b$. All of our functions take nonempty interval arguments and return nonempty interval results except for the intersection operation, which returns an interval which can be empty.

The value of the nonrepresentable constant $\sqrt{3}$ in our algorithm is expanded to a narrow interval. Other constants used are representable. Thus we use the expression $5 H_{o} \leq 1$ rather than $H_{o} \leq 0.2$, which involves the nonrepresentable constant 0.2 . Representable constants such as 5 behave identically in interval arithmetic to intervals where both endpoints have the same value, such as $[5,5]$, and operations involving both intervals and representable constants are treated as if the constant was first converted to a one-point interval.

The verify statement is used to assure that a particular condition holds. If the condition fails, then the entire program is stopped (aborted). If no such failure occurs, the presence of such a verify statement constitutes a proof that the condition holds for the given inputs. One use of the verify statement is in the procedure avgwt defined below, which gives a weighted average of two intervals. To apply this we want to be sure that, as we expect in the cases where it is applied, the first interval is strictly smaller than the second. We check that this is so with a verify statement. Running our program results in no violations of any statement which is tested with a verify statement.

The program examines the set of all torus bubbles to see if any can be a minimizer. A range of hypothetical torus bubbles is specified by intervals $\Theta_{1}$ and $H_{o}$, according to Proposition 4.13. To avoid unnecessary use of trigonometric functions our algorithm uses an equivalent parameterization of the space of torus bubbles by intervals $Y_{1}$ and $H_{o}$, where $Y_{1}=\sin \Theta_{1}$. Rather than solve the mean curvature differential equation directly to find intervals $\left(X_{2}, Y_{2}\right)$ containing the point $\left(x_{2}, y_{2}\right)$ where $\tau_{i}$ and $\tau_{o}$ intersect, we assume existence of a torus bubble, derive $Y_{2}$, and then deduce $X_{2}$ from numerical integrals. Volume calculations for $V_{i}$ and $V_{o}$ are obtained from additional numerical integrals. At each stage in the calculation, we check whether the torus bubbles can be rejected based on the instability results of Section 4. If not, we test whether the volumes are equal. As it turns out all torus bubbles are rejected for these reasons, so area calculations are not necessary and are not present in the algorithm we present. Most torus bubbles are rejected either because $\Theta_{2}$ is out of range, or because the $x$-displacement of $\tau_{i}$ and $\tau_{o}$ differ, or because the volumes enclosed in the two regions differ.

The computation of $\Theta_{2}$ and $Y_{2}$ is based on an analysis of the force function. The integration process used to calculate the value of $X_{2}$ is complicated by the 
fact that the curves may not be graphs, so we have to choose an appropriate parameterization. The most convenient parameterization is in terms of $y$, because the ODEs satisfied by Delaunay curves involve only $y$, and because the $y$-coordinates of the endpoints are specified by $\Theta_{1}$ and $\Theta_{2}$. This allows $x$-displacement and volume to be expressed directly as integrals in terms of $y$, making it unnecessary to actually generate an ODE solution which produces $\tau_{i}$ and $\tau_{o}$.

The curves $\tau_{i}$ and $\tau_{o}$ are generally not graphs as functions of $y$, so the integrals we use have singularities at points where $\dot{y}=0$. A change of variables resolves the singularity, as worked out in Proposition 3.7.

Another difficulty is that the geometry sometimes degenerates at the boundary of the regions we are examining. For example, we need to exclude torus bubbles with $\theta_{1}$ arbitrarily close to 0 , but some of our formulas become singular when $\theta_{1}=0$, and don't make sense there. If the interval $\Theta_{1}$ contains 0 , then various intervals representing $y$-coordinates along the Delaunay curves will also contain 0 , and the integrals give results which will not allow torus bubbles to be eliminated. We get around this problem by using crude estimates which apply in a small zone near the $x$-axis. Near the $x$-axis we have some information about the initial angle and the concavity of the Delaunay curves, and this suffices to give crude bounds to their behavior near the axis. We compute integrals only when at an appropriate distance above the $x$-axis. This approach suffices for all cases except for when $h_{o}$ is also near 0 . In that case we rely on Proposition 4.21.

We now describe in pseudo-code the main interval procedures used in our algorithm. As noted above, aside from the procedures described in this section our algorithm makes use only of standard arithmetic operations on intervals, namely addition, subtraction, multiplication, division, absolute value, maximum, minimum and square.

The procedure avgwt calculates a number in between two inputs, used later to subdivide intervals. It returns a representable real number which is a weighted average. For convenience, we set the rounding mode to round to nearest in this procedure. In some applications of this procedure, an interval is assigned the output of the avgwt procedure, eg in Step 7 of the procedure DivideAndCheckRectangle. The resulting interval then consists of a single point.

real procedure avgwt

input: $\mathrm{X}, \mathrm{Y}, \mathrm{W}$

$$
\begin{aligned}
& \text { verify } X<Y \\
& z:=(1-w) \bar{X}+w \underline{Y} \\
& \text { return } z
\end{aligned}
$$


The interval procedure $\mathbf{S q r t}(\mathbf{X})$ returns an interval containing $\{\sqrt{x}: x \in X$ and $x \geq 0\}$. The real procedure width calculates an upper bound for an interval's width.

The interval procedure Compare checks whether the interval $X$ is to the left of the interval $Y$, returning $A$ if yes, $B$ if $X$ is to the right of $Y$, and the union $A \varpi B$ if the intervals overlap.

interval procedure Compare

input: $\quad X, Y, A, B$

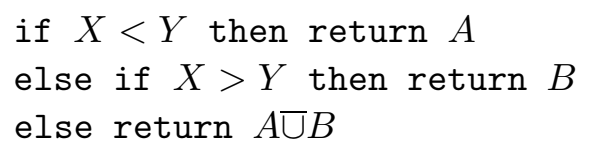

Lemma 5.1. For any $x, y, a, b$, in any $X, Y, A, B$, respectively, if $x \leq y$ then $a \in \mathbf{C o m p a r e}(X, Y, A, B)$, and if $x \geq y$ then $b \in \operatorname{Compare}(X, Y, A, B)$.

Proof. The lemma follows from a straightforward case by case analysis.

Integrate is the basic method for numerical integrals. It gives relatively wide intervals, but is good enough for our purposes. Its input is an intervalvalued function of one interval variable, and two intervals which give the limits of integration.

interval procedure Integrate

input: $F, A, B$

$$
\begin{aligned}
& R:=0 \\
& H:=(\underline{B}-\bar{A}) / 32 \\
& \text { if }(\bar{H}>0) \text { then } \\
& \quad \text { begin } \\
& \quad \text { for } i:=0 \text { to } 31 \text { do } \\
& \quad R:=R+F(\bar{A}+(0 \varpi H)+i H) \\
& \quad \text { end } \\
& \text { return } 0 \bar{\cup} F(A) W i d t h(A)+0 \bar{\cup} F(B) W i d t h(B)+R H
\end{aligned}
$$

Proposition 5.2. For any integrable $f$ and real numbers $a, b$, with $a \leq b$, and interval extensions $F, A, B$, the integral of $f$ from $a$ to $b$ is contained in Integrate $(F, A, B)$.

Proof. If $A \leq B$,

$$
\int_{a}^{b} f d x=\int_{a}^{\bar{A}} f d x+\int_{\bar{A}}^{\underline{B}} f d x+\int_{\underline{B}}^{b} f d x .
$$


The term $\int_{a}^{\bar{A}} f d x$ is contained in $0 \bar{\cup} F(A) \mathrm{Width}(A)$ and similarly $\int_{\underline{B}}^{b} f d x$ is contained in $0 \cup F(B) \mathrm{Width}(B)$. Note that we need to replace $F(\bar{A})$ and $F(B)$ by $0 \varpi F(A)$ and $0 \varpi F(B)$ to deal with the issue that $a$ can lie anywhere in $A$ and similarly $b$ in $B$. Taking the union of $F$ with 0 ensures that our sum contains the intervals $F([a, \bar{A}])$ and $F([\underline{B}, b])$.

The value of $\int_{\bar{A}}^{\underline{B}} f d x$ is bounded by lower and upper Riemann sums, using 32 equally sized intervals. The term $F(\bar{A}+(0 \bar{\cup})+i H)$ evaluates $F$ on an interval which contains $[\bar{A}+i h, \bar{A}+(i+1) h]$ for any $h \in H$, and so contains both an upper and a lower bound for $F$ on this interval. The $R H$ term in the last line, when added to the previous terms, then gives an interval containing both lower and upper Riemann sums for $\int_{a}^{b} f d x$.

Otherwise, $A \leq B$ is false, and since $a \leq b, A$ and $B$ must overlap, so we can decompose

$$
\int_{a}^{b} f d x=\int_{a}^{c} f d x+\int_{c}^{b} f d x
$$

where $a \leq c \leq b$ and $c \in A \cap B$. Since $\underline{B}<\bar{A}, R=0$ and the $R H$ term in the last line vanishes. The result follows from $[a, c] \subset[a, \bar{A}],[c, b] \subset[\underline{B}, b]$ and inclusion monotonicity.

The next several procedures define functions to be integrated.

The procedure $\mathbf{D x}$ is a straightforward interval extension of the expression for $d x / d y$ given in equation 6 in Proposition 3.6. It will be used to find the $x$ displacement from $\left(x_{1}, y_{1}\right)$ to $\left(x_{2}, y_{2}\right)$ by applying equation 6 to both $\tau_{i}$ and $\tau_{o}$.

Volumes under Delaunay surfaces are computed using the volume integrand Dv, which is obtained from $\mathbf{D x}$ as in Proposition 3.6. It is convenient to have Dv calculate volume divided by $\pi$. Since volumes are only compared to one another, we can avoid the unnecessary step of multiplying by the nonrepresentable constant $\pi$. The volume element computation required that $d x / d y>0$ for the volume to have a positive sign. The curve $\tau_{o}$ will sometimes start out in the negative $x$ direction, when $\theta_{1}<60^{\circ}$, and after passing through a vertical tangency switch to going in the positive $x$ direction. Calculating the integral of Dv in this case will give the volume under the part of the nodoid generated by the arc going right minus the volume under the arc going left. This is just what we want to compute the volume $V_{o}$ in the torus component in the case when such an overhang exists.

At local minima and maxima, $\mathbf{D x}$ and $\mathbf{D v}$ are singular. $Y_{\min }$ and $Y_{\max }$ are global variables which are accessed by $\mathrm{Dx}_{\min }, \mathrm{Dx}_{\max }, \mathrm{Dv}_{\min }$ and $\mathrm{Dv}_{\max }$. So near local extrema, we use a change of variables to reparameterize the curve, as in Proposition 3.7. Procedure $\mathbf{D} \mathbf{x}_{\min }$ calculates $d x / d z$ near a minimum 
and procedure $\mathbf{D} \mathbf{x}_{\max }$ near a maximum, where $z$ is given in Proposition 3.7. We do an algebraic manipulation to make the denominator in the square root more computationally effective, namely we replace the term $H Y-2+H Y_{\max }$ in procedure $\mathrm{Dx}_{\max }$ by the equivalent expression $H\left(Y+Y_{\text {outer min }}\right)$. Here $Y_{\text {outer min }}$ represents the minimum $y$-value of the nodoid which has a maximum at $Y_{\max }$. The equivalence of the two expressions is derived using Proposition 3.4 as follows:

$$
H Y-2+H Y_{\max }=\frac{2}{Y_{\max }-Y_{\text {outermin }}}\left(Y+Y_{\max }\right)-2=H\left(Y+Y_{\text {outermin }}\right) .
$$

The volume calculation procedures $\mathbf{D} \mathbf{v}_{\min }, \mathbf{D} \mathbf{v}_{\max }$ give the formula for the volume integrand used near a critical point. The volume calculations are applied only to graphs over the $x$-axis.

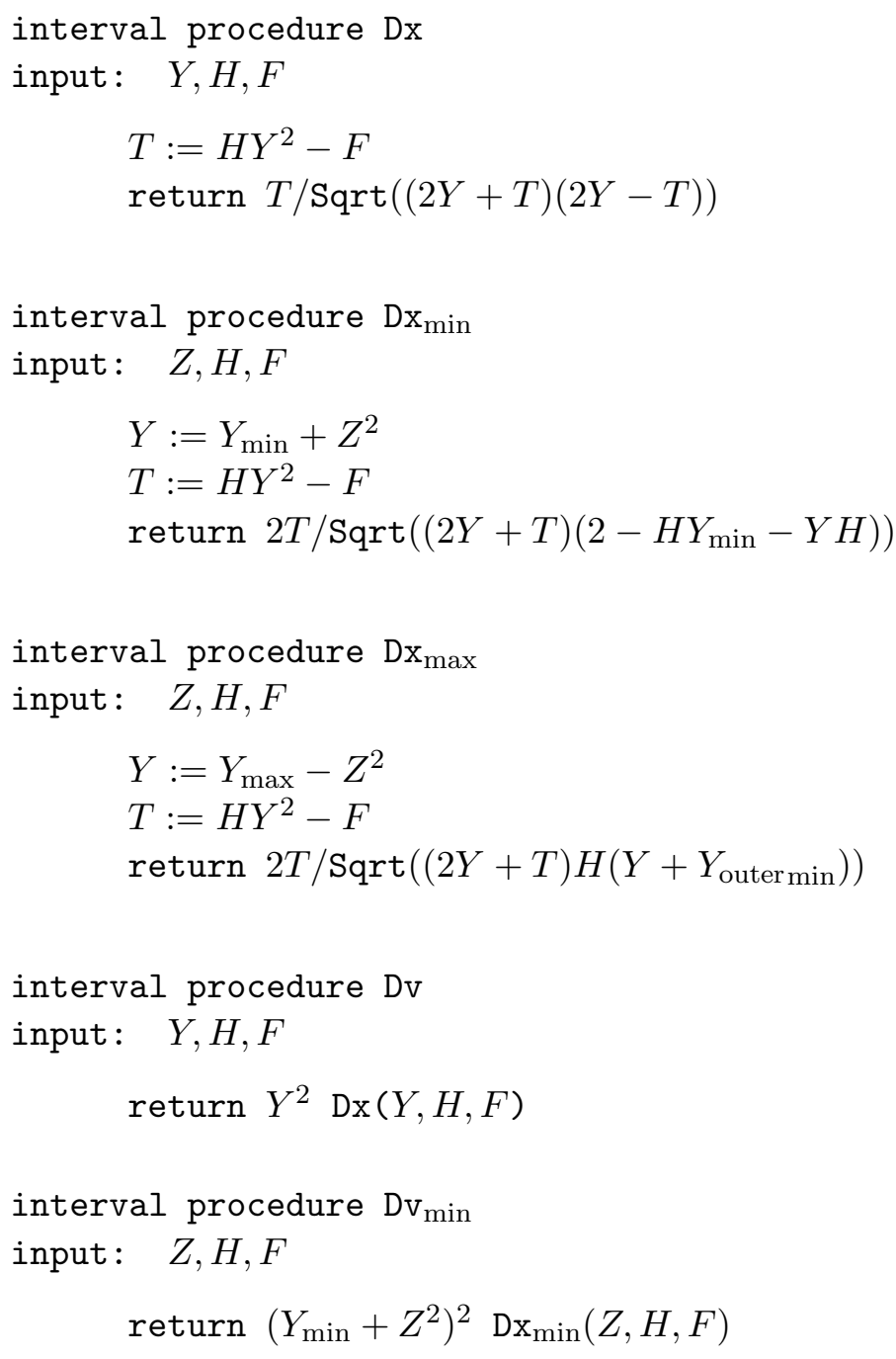


interval procedure $\mathrm{Dv}_{\max }$

input: $Z, H, F$

return $\left(Y_{\max }-Z^{2}\right)^{2} \operatorname{Dx}_{\max }(Z, H, F)$

The next procedure is used for testing interval rectangles of torus bubbles to see if they are potential minimizers and rejecting these intervals if they can be shown to have a property which rules out any torus bubble contained in them. The input is a pair of intervals $C_{1}$ and $H_{o}$, which give a range of possible values for $\cos \theta_{1}$ and $h_{o}$, respectively. The interval of cosine values $C_{1}$ is used, rather than an interval of $\theta_{1}$ values, to eliminate calls to trigonometric functions. A variety of tests are performed based on the analysis of previous sections. The various intervals do not necessarily give sharp estimates, so it is not a priori clear how many rectangles will be rejected. As it turns out, the tests are sharp enough to reject all rectangles.

Boolean procedure CheckRectangle

input: $C_{1}, H_{O}$

1. if $1000 C_{1} \geq 996$ and $5 H_{o} \leq 1$

then return REJECT

2. $H_{i}:=2-H_{o}$

$Y_{1}:=\operatorname{Sqrt}\left(1-C_{1}^{2}\right)$

$F_{i}:=\left(H_{i}-1\right) Y_{1}^{2}-C_{1} Y_{1} \operatorname{Sqrt}(3)$

$F_{o}:=-F_{i}$

$C_{2}:=\left[-C_{1} \varpi C_{1}\right] \cap[-.5 \cup .5]$

$T:=\left(2\left(H_{i}-1\right) F_{i}+3\right) /\left(3+\left(H_{i}-1\right)^{2}\right)-\left(1-C_{1}^{2}\right)$

if $\left(C_{2}^{2}+T\right) \neq 1$ return $\mathrm{REJECT}$

3. $Y_{2}:=\operatorname{Sqrt}(T \cap[0,1])$

$C_{2}:=C_{2} \cap \frac{\left(H_{i}-1\right) Y_{2}-F_{i} / Y_{2}}{\operatorname{Sqrt}(3)}$

if $C_{2}$ is empty then return REJECT

4. if $C_{1} \leq .5$ and $H_{o} \leq 1-\operatorname{Sqrt}(3) C_{1} / Y_{1}$

then return REJECT

5. if $\operatorname{Width}\left(C_{2}\right)>.5$ then return NORESULT

6. $W_{\text {ends }}:=\left(1-C_{1}\right)^{2}\left(2+C_{1}\right) / 3+\left(1-C_{2}\right)^{2}\left(2+C_{2}\right) / 3$

$Y_{\min }:=-F_{i} /\left(1+\operatorname{Sqrt}\left(1+F_{i} H_{i}\right)\right)$

$Y_{\max }:=\left(1+\operatorname{Sqrt}\left(1+F_{o} H_{o}\right)\right) / H_{o}$ 


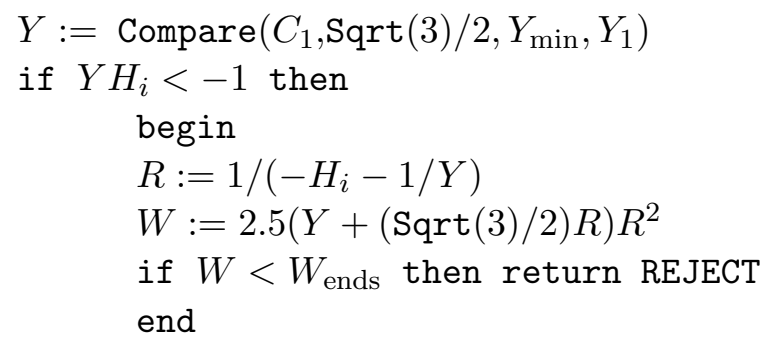

7. $\quad Y_{\text {left }}:=\operatorname{Sqrt}\left(F_{o} / H_{o}\right)$

if $\left(Y_{\min }<Y_{2}\right.$ and $\left.Y_{\text {left }}<Y_{\max }\right)$ then $Y_{\text {left }}:=\operatorname{Max}\left(Y_{1}, Y_{\text {left }}\right)$ else return NORESULT

$Y_{4}:=\operatorname{avgwt}\left(Y_{\text {left }}, Y_{\max }, .5\right)$

$Z_{2}:=\operatorname{Sqrt}\left(Y_{\max }-Y_{2}\right)$

$Z_{4}:=-\operatorname{Sqrt}\left(Y_{\max }-Y_{4}\right)$

if $1000 C_{1} \geq 998$ then

$$
\begin{aligned}
& \text { begin } \\
& T:=\operatorname{avgwt}\left(Y_{1}, Y_{2}, 1 / 16\right) \\
& \Delta_{i}:=\left(T-Y_{1}\right)(33 / 16)+\operatorname{Integrate}\left(\operatorname{Dx}\left(\cdot, H_{i}, F_{i}\right), T, Y_{2}\right) \\
& \Delta_{o}:=-\left(Y_{\text {left }}-Y_{1}\right) \operatorname{Sqrt}(3) \\
& T:=\operatorname{avgwt}\left(Y_{\text {left }}, Y_{4}, 1 / 16\right) \\
& \Delta_{o}:=\Delta_{o}+\operatorname{Integrate}\left(\operatorname{Dx}\left(\cdot, H_{o}, F_{o}\right), T, Y_{4}\right) \\
& \text { if } \Delta_{o}>\Delta_{i} \text { then return } \operatorname{REJECT} \\
& \Delta_{o}:=\Delta_{o}+\text { Integrate }\left(\operatorname{Dx} \max \left(\cdot, H_{o}, F_{o}\right), Z_{4}, Z_{2}\right) \\
& \text { if } \Delta_{o}>\Delta_{i} \text { then return } \operatorname{REJECT} \\
& \text { if } 1 \in C_{1} \text { then return NORESULT } \\
& \text { end }
\end{aligned}
$$$$
\Delta_{o}:=\Delta_{o}+\text { Integrate }\left(\mathrm{Dx}_{\max }\left(\cdot, H_{o}, F_{o}\right), Z_{4}, Z_{2}\right)
$$

8. verify $\left(C_{1} \leq \operatorname{Sqrt}(3) / 2\right.$ or $\left.Y_{1} \leq Y_{2}\right)$

$T:=\operatorname{Sqrt}\left(Y_{1}-Y_{\min }\right)$

$Z_{1}:=$ Compare $\left(C_{1}, \operatorname{Sqrt}(3) / 2,-T, T\right)$

$Z_{3}:=\operatorname{Sqrt}\left(Y_{2}-Y_{\min }\right)$

$\Delta_{i}:=$ Integrate $\left(\mathrm{Dx}_{\min }\left(\cdot, H_{i}, F_{i}\right), Z_{1}, Z_{3}\right)$

$\Delta_{o}:=$ Integrate $\left(\mathrm{Dx}\left(\cdot, H_{o}, F_{o}\right), Y_{1}, Y_{4}\right)$

if $\Delta_{i}<\Delta_{o}$ then return REJECT

$\Delta_{o}:=\Delta_{o}+$ Integrate $\left(\mathrm{Dx}_{\max }\left(\cdot, H_{o}, F_{o}\right), Z_{4}, Z_{2}\right)$

if $\Delta_{i} \neq \Delta_{o}$ then return REJECT

9. $\quad W_{\text {base }}:=$ Integrate $\left(\operatorname{Dv}_{\min }\left(\cdot, H_{i}, F_{i}\right), Z_{1}, Z_{3}\right)$

$W_{i}:=W_{\text {ends }}+W_{\text {base }}$

$W_{o}:=$ Integrate $\left(\operatorname{Dv}\left(\cdot, H_{o}, F_{o}\right), Y_{1}, Y_{4}\right)+$

Integrate $\left(\mathrm{Dv}_{\max }\left(\cdot, H_{o}, F_{o}\right), Z_{4}, Z_{2}\right)-W_{\text {base }}$ 
if $W_{i} \neq W_{o}$ then return REJECT

else return NORESULT

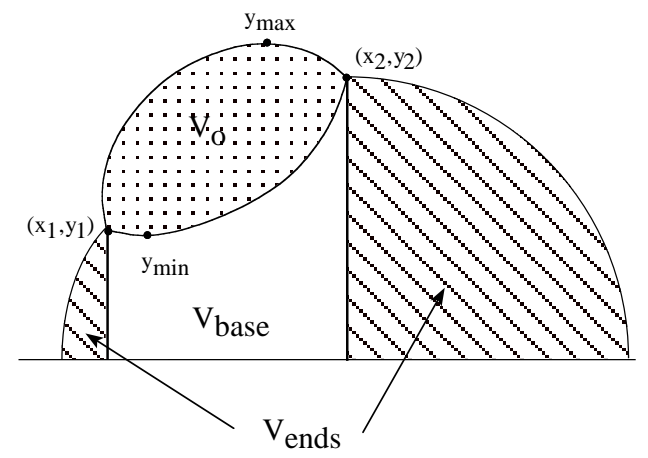

Figure 17. Compared volumes. If we can show that $V_{o}$ is not equal to $V_{\text {base }}+V_{\text {ends }}$ then we can discard the torus bubble as a potential equal volume minimizer.

TheOREM 5.3. If CheckRectangle $\left(C_{1}, H_{o}\right)$ returns REJECT without causing an IEEE exception, then there is no area-minimizing equal-volume torus bubble with $\cos \theta_{1} \in C_{1}$ and $h_{o} \in H_{o}$.

Proof. The procedure CheckRectangle is fed a pair of intervals containing a range of values for $\cos \theta_{1}$ and $h_{o}$, these intervals being denoted by $C_{1}$ and $H_{o}$. We will call such a range of input values an input rectangle. If every pair of values $\left(\cos \theta_{1}, h_{o}\right) \in C_{1} \times H_{o}$ in an input rectangle can be ruled out as a possible minimizing torus bubble by some test, then the procedure returns the value REJECT. Otherwise it returns the value NORESULT (which results in a subdivision of the input rectangle into four smaller rectangles.) The tests applied to the rectangles of values for $\cos \theta_{1}$ and $h_{o}$ are based on the results developed in this paper, as we will explain below. At certain points we need to check whether some hypothesis required in our tests are satisfied. We can do so by using a verify statement. This statement will cause the program to terminate if it is not satisfied. The proof proceeds by interpreting and justifying each step of the algorithm.

Step 1 rejects torus bubbles based on Proposition 4.21. $C_{1}$ is a range of cosine values for the angle $\theta_{1}$, so $1000 C_{1} \geq 996$ corresponds to $\cos \theta_{1} \geq .996$, which implies that $\theta_{1} \leq 5.2^{\circ}$ and the proposition applies.

Step 2 first defines some values used in further steps. The values of $F_{o}$ and $F_{i}$ are calculated using the formulas in Proposition 4.16. It then examines the possible values of $y_{2}=\sin \theta_{2}$. Corollary 4.10 implies $-.5 \leq \cos \theta_{2}$, and Lemma 4.8 implies $\cos \theta_{2} \leq .5$. Since we assumed that $\theta_{1} \leq \theta_{2}$, we have 
$\cos \theta_{2} \leq \cos \theta_{1}$. Proposition 4.14 implies that $-\cos \theta_{1} \leq \cos \theta_{2}$. Thus the values for $\cos \theta_{2}$ arising from possible minimizing torus bubbles lie in the intersection of the intervals $[-.5, .5]$ and $\left[-\cos \theta_{1}, \cos \theta_{1}\right]$.

The force equation for $T_{i}$,

$$
f_{i}(c)=\left(h_{i}-1\right)\left(1-c^{2}\right)-c \sqrt{3\left(1-c^{2}\right)},
$$

has at most two roots by Lemma 4.15 , one of which is $c_{1}$. The other one is $c_{2}$. Letting $y=\sqrt{1-c^{2}}$ and rearranging gives

$$
\begin{array}{r}
f_{i}=\left(h_{i}-1\right) y^{2}+c y \sqrt{3} \\
\left(f_{i}-\left(h_{i}-1\right) y^{2}\right)^{2}=3 y^{2}\left(1-y^{2}\right) .
\end{array}
$$

This is a quadratic in $y^{2}$. The sum of the two roots in $y^{2}$ is

$$
\frac{2\left(h_{i}-1\right) f_{i}+3}{3+\left(h_{i}-1\right)^{2}}
$$

and the known root is $1-c_{1}^{2}$, so subtraction gives the other root. Thus the interval $T$ contains $y_{2}^{2}$, the square of the second root. We next add the interval $C_{2}^{2}$, containing $\cos ^{2} \theta_{2}$, to the interval $T$ containing $\sin ^{2} \theta_{2}$ to see if they contain values adding to one. If not, there is no minimizing torus bubble in the chosen input rectangle $C_{1} \times H_{o}$, and this input rectangle is rejected.

Step 3 calculates the possible values of $x_{2}=\cos \theta_{2}$ corresponding to $y$-values whose square is in the interval $T$. The only possible values for $y_{2}$ are in $[0,1]$, so we intersect $T$ with $[0,1]$ before taking the square root of $T$ to get an interval $Y_{2}$ which contains possible values of $y_{2}$. Since

$$
f_{i}=\left(h_{i}-1\right)\left(1-c_{2}^{2}\right)-c_{2} \sqrt{3\left(1-c_{2}^{2}\right)}
$$

by Proposition 4.16 , and $1-c_{2}{ }^{2}=y_{2}^{2}$, we can solve to get an expression for $c_{2}$ in terms of $h_{i}, f_{i}$ and $y_{2}$ :

$$
C_{2}=\left(\left(H_{i}-1\right) Y_{2}-F_{i} / Y_{2}\right) / \operatorname{Sqrt}(3)
$$

Note that this expression is chosen to give us $c_{2}$ with the correct sign. We intersect the resulting interval with the interval $C_{2}$ computed in the previous step. If the resulting interval is empty, there is no minimizing torus bubble in the chosen input rectangle and it is rejected.

Step 4 rejects torus bubbles based on Proposition 4.18. It uses the fact that $\cot \left(\theta_{1}\right)=c_{1} / y_{1}$.

Step 5 passes on a request to subdivide the rectangle if it is too wide. It does not reject anything, so there is nothing to justify. This step is included to speed the program's execution. 
Step 6 calculates rough bounds to check if the volumes of the two regions can be equal. We calculate volume divided by $\pi$ in the algorithm to avoid the needless step of multiplying by the nonrepresentable constant $\pi$. The interval $W_{\text {ends }}=V_{\text {ends }} / \pi$ is defined to be the volume of the solid of revolution between the spherical ends and the $x$-axis, divided by $\pi$. See Figure 17 . This solid is wholly contained in the ball component. $Y_{\min }$ contains the $y$-coordinate of the local minimum of the $\tau_{i}$, based on solving the force equation as in Part 7 of Proposition 4.12. This minimum is realized in the torus bubble if and only if $\theta_{1} \geq 30^{\circ}$. Similarly $Y_{\max }$ contains the maximum height for $\tau_{o}$, as in Part 8 of Proposition 4.12. It is always achieved. Step 6 goes on to reject potential torus bubbles if a calculation shows that the torus component has less than half the total volume. The volume of the ball component is bounded below by the volume under the spherical caps, whose value is given by $V_{\text {ends }}$. The interval $Y$ is defined to ensure that it contains the minimal $y$-coordinate of the torus component, which occurs either in $Y_{\min }\left(\right.$ if $\theta_{1} \geq 30^{\circ}$ ) or in $Y_{1}$. An upper bound $V$ for the torus component volume is calculated using the formula derived in Lemma 4.24.

Step 7 tries to eliminate torus bubbles with small $\theta_{1}$, namely those with $\cos \theta_{1} \geq .998$, a condition which forces $\theta_{1}$ to be less than $3.7^{\circ}$ and allows Lemma 4.26 to be applied. These small angles must be treated differently because the equations describing Delaunay curves are not well behaved near $\theta_{1}=0$. Bounds on the $x$-displacement $x_{2}-x_{1}$ are calculated using each of $\tau_{i}$ and $\tau_{o}$. The interval $\Delta_{i}$ contains an upper bound for the width of $\tau_{i}$ and the interval $\Delta_{o}$ contains a lower bound for the width of $\tau_{o}$. If the resulting intervals satisfy $\Delta_{i}<\Delta_{o}$ then $\tau_{i}$ does not travel as far to the right as $\tau_{o}$ and the input rectangle can be rejected.

Step 7 begins by defining $Y_{\text {left }}$ to be an interval containing the $y$-value of the point where $\tau_{o}$ has a vertical tangency on the left as in Figures 18 and 19. This value is computed by taking the square root of the nonnegative part of $F_{o} / H_{o}$. Corollary 3.3 guarantees that this value is positive in a torus bubble, so we are justified in truncating negative values which may have appeared in the calculation before taking the square root. The outer curve is always a nodoid by Corollary 4.19, and if extended to a vertical tangency, the $y$-coordinate of that point is in the interval $Y_{\text {left }}$. Replacing $Y_{\text {left }}$ with $\operatorname{Max}\left(Y_{\text {left }}, Y_{1}\right)$ assures that $Y_{\text {left }}$ contains the $y$-coordinate of a vertical tangency if there is one, and the initial point otherwise. In particular it always contains the $y$-coordinate of the leftmost point.

The value $Y_{4}$ is used as an intermediate point for chopping up the intervals of integration for $\tau_{o}$ between its vertical and horizontal tangencies. See Figures 18, 19, 20. The limits of integration $Z_{2}$ and $Z_{4}$ are defined for use when integrating near a maximum of $\tau_{o}$, as in Proposition 3.7. The square 
roots taken in these calculations are justified in discarding the negative part of any interval, because for any particular torus bubble the quantity whose root is being taken is nonnegative.

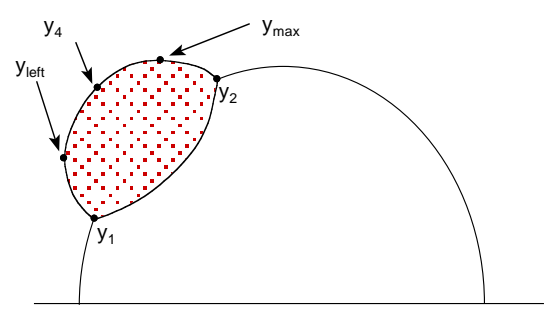

Figure 18. A double bubble configuration for $0<\theta_{1} \leq 30$.

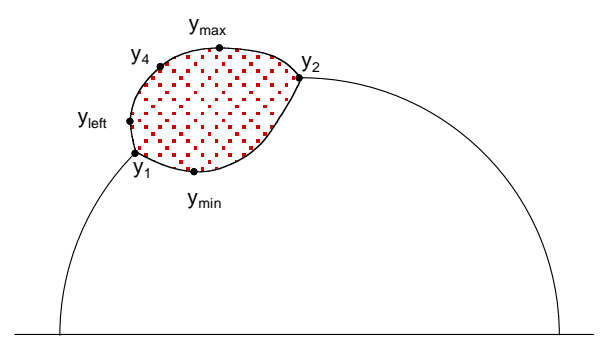

Figure 19. A double bubble configuration for $30 \leq \theta_{1} \leq 60$.

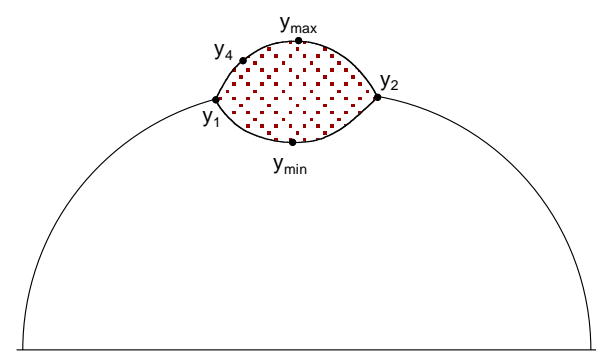

Figure 20. A double bubble configuration for $60 \leq \theta_{1} \leq 90$.

An upper bound for the $x$-displacement of $\tau_{i}$ as it goes from $y_{1}$ to $y_{2}$ is given by $\Delta_{i}$, and a lower bound for the $x$-displacement of $\tau_{o}$ as it goes from $y_{1}$ to $y_{2}$ is given by $\Delta_{o}$. If $\Delta_{i}<\Delta_{o}$, then a torus bubble cannot occur in the given input rectangle.

We use the Intermediate Value Theorem to estimate $\Delta_{i}$ up to height $y_{1}+$ $\frac{y_{2}-y_{1}}{16}$ and $\Delta_{o}$ up to height $y_{\text {left }}$. The estimates for $\Delta_{i}$ and $\Delta_{o}$ near the $x$-axis are justified by Proposition 3.4 in the case of $\tau_{o}$ and by Lemma 4.26 in the case of $\tau_{i}$, as shown in Figure 21. 


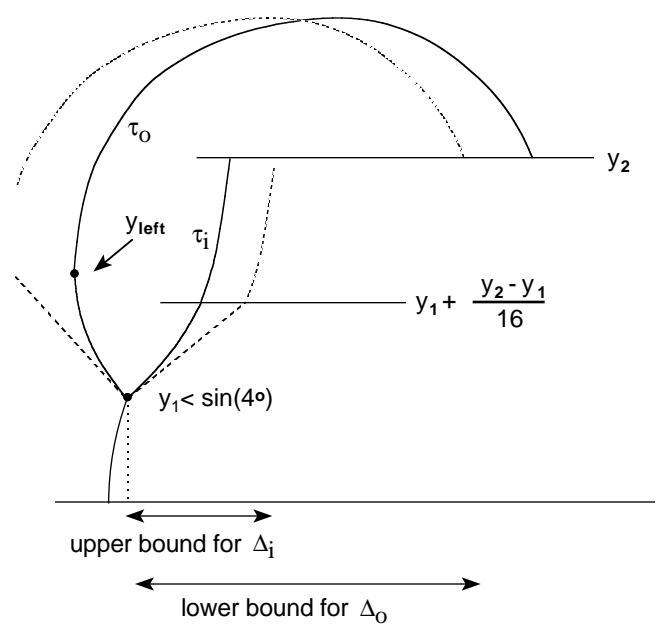

Figure 21. Estimating the widths of $\tau_{i}$ from above and $\tau_{o}$ from below with $\theta_{1}$ small.

For $\theta_{1}<4^{\circ}, \tau_{i}$ is strictly increasing, so that the $x$-displacement is equal to the integral of $\mathbf{D x}$ from $y_{1}$ to $y_{2}$. This can be broken up into an integral from $Y_{1}$ to $T$ and an integral from $T$ to $Y_{2}$, where $T$ is set to be one sixteenth of the way from $Y_{1}$ to $Y_{2}$. The values in $T$ are all less than $1 / 2$, so that Lemma 4.26 applies and the slope of $\tau_{i}$ below height $T$ is smallest at its initial point $\left(x_{1}, y_{1}\right)$. It follows that

$$
d x / d y \leq \cot \left(30-\theta_{1}\right) \leq \cot 26^{\circ}<33 / 16
$$

on this interval and the integral from $Y_{1}$ to $T$ is bounded above by $(33 / 16)$ $\left(T-Y_{1}\right)$. Then $\Delta_{i}=\left(T-Y_{1}\right)(33 / 16)+\operatorname{Integrate}\left(\mathrm{Dx}\left(\cdot, H_{i}, F_{i}\right), T, Y_{2}\right)$ gives an upper bound for the width of $\tau_{i}$ as it increases from $Y_{1}$ to $Y_{2}$.

With $\tau_{o}$ we first find an upper bound on the leftward displacement between $y_{1}$ and $y_{\text {left }}$ using a similar method. In this case the slope has absolute value larger than $\tan 30^{\circ}=1 / \sqrt{3}$ and $\left(Y_{\text {left }}-Y_{1}\right) \operatorname{Sqrt}(3)$ gives an upper bound for the leftward displacement to height $y_{\text {left }}$. An integral is calculated which gives a lower bound for the rightwards displacement of $\tau_{o}$ between $y_{\text {left }}$ and $y_{2}$, by integrating $d x / d y$ from a point strictly above $y_{\text {left }}$ to $y_{2}$. This integral is calculated in two pieces, the first to $Y_{4}$ and the second from $Y_{4}$ through the local maximum to $Y_{2}$. A lower bound for $\Delta_{o}$ is gotten by taking the upper bound for the leftwards displacement previously calculated and subtracting it from the sum of these integrals. If $\Delta_{o}>\Delta_{i}$ the input rectangle is rejected.

This step can reject torus bubbles even if the interval $C_{1}$ contains the value 1 , corresponding to $\theta_{1}=0$. If this step test fails to reject a range of potential torus bubbles, we check whether 1 is in the interval $C_{1}$. If it is, then the next steps will not help, since they are ineffective for $\theta_{1}=0$. So if 1 is 
in $C_{1}$ we return NORESULT and send the current input rectangle back for subdivision.

Step 8 calculates intervals for the $x$-displacement of $\tau_{i}$ and $\tau_{o}$ in the case where the angle is larger than those treated in the previous step, namely $\cos \theta_{1} \leq .996$. In this step $\Delta_{o}$ is an interval containing the $x$-displacement of the outer curve and $\Delta_{i}$ an interval containing the $x$-displacement of $\tau_{i}$. In contrast to Step 7, they do not contain bounds for these displacements, but the actual widths of $\tau_{i}$ and $\tau_{o}$. Thus we can reject the input rectangle in this step if $\Delta_{i}$ and $\Delta_{o}$ do not overlap.

The interval $\Delta_{o}$ is calculated by integrating $\mathbf{D x}$ from $Y_{1}$ to an intermediate $Y_{4}$ and then using $\mathbf{D} \mathbf{x}_{\max }$ to integrate from $Y_{4}$ across a maximum to $Y_{2}$. If the width of $\Delta_{o}$ is larger than 20 then the procedure returns NORESULT, calling for a subdivision of the input rectangle of intervals. This is done because otherwise large amounts of computational time are wasted in inefficient calculations.

The calculation of $\Delta_{i}$ is complicated somewhat because we need to deal with both the case where $\theta_{1} \geq 30^{\circ}$ and the case where $\theta_{1} \leq 30^{\circ}$. Only in the former case, where $C_{1} \leq \operatorname{Sqrt}(3) / 2$, does $\tau_{i}$ achieve an interior minimum. In that case, an integral along $\tau_{i}$ can be calculated by integrating $\mathbf{D} \mathbf{x}_{\min }$ from $Z_{1}$ to $Z_{3}$. If an interior minimum is not achieved, we can still integrate $\mathbf{D} \mathbf{x}_{\min }$ to calculate the $x$-displacement from $Y_{1}$ to $Y_{2}$, but we need to reverse the sign of $Z_{1}$ as in the last statement of Proposition 3.7. Our integral formulas for $x$-displacement need to have that $Y_{1} \leq Y_{2}$ in this case, so we check with a verify statement that either this holds or there is an interior minimum. The Compare statement ensures the correct sign is given to $Z_{1}$ unless $C_{1}$ overlaps the interval $\operatorname{Sqrt}(3) / 2$. $Z_{1}$ equals -Sqrt $\left(Y_{1}-Y_{\min }\right)$ if $\tau_{i}$ achieves an interior minimum and $\operatorname{Sqrt}\left(Y_{1}-Y_{\min }\right)$ otherwise. The appropriate sign is given to $Z_{1}$ using a Compare statement. If $C_{1}$ overlaps the interval $\operatorname{Sqrt}(3) / 2$ we can't tell which sign is correct, so the Compare statement returns an interval containing both $Z_{1}$ and $-Z_{1}$, and the integral then contains the $x$-displacements for both cases. If $\Delta_{i}$ and $\Delta_{o}$ are disjoint, then the curves' final points cannot coincide, and the input rectangle is discarded.

Step 9 calculates volumes divided by $\pi$, as in Figure 17. We again calculate volume divided by $\pi$ in the algorithm to avoid an unnecessary multiplication of all volumes by $\pi$. $V_{\text {base }}$ is the volume surrounded by $T_{i}$ and $W_{\text {base }}=V_{\text {base }} / \pi$. Adding $W_{\text {base }}$ to $W_{\text {ends }}$ gives $\left.W_{i}=V_{i} / \pi\right)$. The calculation of $V_{o}$ is slightly more complicated because the overhang on the left involves a subtraction, if there is an overhang. Such an overhang occurs when $\theta_{1}<60^{\circ}$, as shown in Figures 18 and 19. The formulas are set up so that volume is counted with a negative sign when $\tau_{o}$ is oriented to the left and positive sign when $\tau_{o}$ is oriented to the right, as discussed in Proposition 3.6. This is just what is required to calculate the volume of the overhang. The volume $V_{\text {base }}$ is subtracted from the volume 
inside $T_{o}$ to get $V_{o}$, the volume of the torus component. Step 9 then rejects an input rectangle if the values $W_{o}=V_{o} / \pi$ and $W_{i}=V_{i} / \pi$ are unequal.

Now that we have tests that are able to reject certain ranges of torus bubbles, we apply these tests to see whether they in fact reject all the possibilities. Since we don't know in advance how fat the ranges can be, we initially feed in a rectangle of values with $0 \leq \sin \theta_{1} \leq 1,0 \leq h_{o} \leq 10$. We recursively subdivide further as necessary. We monitor IEEE exception flags at this level, so that no computation is trusted if it raised an exception.

Boolean procedure DivideAndCheckRectangle

input: $Y_{1}, H_{i}$

$C_{1}:=\operatorname{Sqrt}\left(1-Y_{1}^{2}\right)$

call CheckRectangle $\left(C_{1}, H_{o}\right)$

if result is REJECT then return SUCCESS

split $Y_{1}$ in half, into $Y_{1 a}, Y_{1 b}$

split $H_{o}$ in half, into $H_{o a}, H_{o b}$

call DivideAndCheckRectangle $\left(Y_{1 a}, H_{o a}\right)$

call DivideAndCheckRectangle $\left(Y_{1 a}, H_{o b}\right)$

call DivideAndCheckRectangle $\left(Y_{1 b}, H_{o a}\right)$

call DivideAndCheckRectangle $\left(Y_{1 b}, H_{o b}\right)$

return SUCCESS

Main program

begin

clear exceptions

begin

$Y_{1}:=[0,1]$

$H_{o}:=[0,10]$

Call DivideAndCheckRectangle $\left(Y_{1}, H_{o}\right)$

end

verify no exceptions raised

Print "All torus bubbles rejected."

end

Theorem 5.4. The algorithm described in Main, if run to completion without causing an exception, shows that no area minimizing torus bubble can enclose equal volumes.

Proof. By Proposition 4.13, any minimizing torus bubble is determined by $\theta_{1}$ and $h_{o}$, where $\theta_{1}$ is the angle of first arc and $h_{o}$ is the mean curvature of $T_{o}$. 
It suffices to consider $0 \leq \theta_{1} \leq 90^{\circ}$ by Proposition 4.14 , and $0 \leq h_{o} \leq 10$ by Proposition 4.3 and Proposition 4.25.

Main calls DivideAndCheckRectangle with a rectangle containing the parameters of all possible torus bubbles. DivideAndCheckRectangle subdivides this rectangle, depending on the success of its calculations. To avoid unnecessary use of trigonometric functions in the computation, the space of angles is parameterized by a variable $y_{1}=\sin \theta_{1}$. Thus the intervals $Y_{1}$ cover the interval $[0,1]$, in $1-1$ correspondence with $0 \leq \theta_{1} \leq 90^{\circ}$. DivideAndCheckRectangle begins by using $Y_{1}$ to compute $C_{1}$, an interval corresponding to values of the cosine of an interval of $\theta_{1}$ angles. It then passes the $C_{1}$ and $H_{o}$ intervals to CheckRectangle where they are tested as previously described.

Main can only finish if CheckRectangle rejects all rectangles. By Theorem 5.3, torus bubbles rejected by CheckRectangle are not minimizers.

We implemented the algorithm described above in $\mathrm{C}++$, though we could equally well have used any language which allows for control of rounding and follows the IEEE 754 standard, or any similar precise prescription for floating point arithmetic. We ran code implementing this algorithm on a variety of machines, various Wintel, Sun, HP, SGI, UNIX and Linux platforms, with identical results. The $\mathrm{C}++$ code with embedded instructions is available at the web site [21]. The code takes about 10 seconds to run on a fast (1999) PC. The input rectangle was recursively subdivided up to ten times, resulting in a total of 15,016 rectangles examined. The calculation involved a total of 51,256 integrals.

From examining the output of the program, we can see the reasons for which various rectangle were rejected. Of the 15,016 rectangles examined, Step 1 rejected 31, Step 2 rejected 1140, Steps 3, 4 and 5 rejected none, Step 6 rejected 3664, Step 7 rejected 541, Step 8 rejected 6691 and Step 9 rejected 2949. In Figure 22 we indicated which steps were used to reject torus bubbles in which input rectangles. This chart offers some insight into why torus bubbles fail to be minimizers. However there is some arbitrariness to this, as doing the steps in a different order would have produced different data.

In running the program no exceptions were raised, and the program returned the statement "All torus bubbles rejected." As a consequence we obtain a proof of our main result.

THEOREM 1. The unique surface of least area enclosing two equal volumes in $\mathbb{R}^{3}$ is a double bubble.

Proof. Theorem 5.4 eliminates the possibility of a minimizing torus bubble. Theorem 4.1 implies that the only other possibility is a double bubble. 


\begin{tabular}{|c|c|c|c|c|c|c|c|c|c|c|c|c|c|c|c|c|}
\hline .9375-1 & 2 & 2 & 2 & 2 & 2,8 & $2,8,9$ & 2,9 & $2,6,9$ & 6,9 & 6 & 6 & 6 & 6 & 6 & 6 & 6 \\
\hline $.875-.9375$ & 2 & 2 & 2 & 2,8 & 2,8 & 8,9 & 9 & 9 & 6,9 & 6 & 6 & 6 & 6 & 6 & 6 & 6 \\
\hline $.8125-875$ & 2 & 2 & $2,8,9$ & 2,8 & 8 & 8,9 & 9 & 9 & 6,9 & 6 & 6 & 6 & 6 & 6 & 6 & 6 \\
\hline $.75-.8125$ & 2 & 2 & $2,8,9$ & 8 & 8 & 8,9 & 9 & 9 & 6,9 & 6 & 6 & 6 & 6 & 6 & 6 & 6 \\
\hline $.6875-75$ & 2 & 2 & $2,8,9$ & 8 & 8 & 8,9 & 9 & 9 & 6,9 & 6 & 6 & 6 & 6 & 6 & 2,6 & 2,6 \\
\hline $.625-6875$ & 2 & 2,8 & $2,8,9$ & 8 & 8 & 8,9 & 9 & 9 & 6,9 & 6 & 2,6 & 2,6 & 2,6 & 2,6 & 2,6 & 2 \\
\hline $.5625-.625$ & 2 & 2,8 & 8 & 8 & 8 & 8,9 & 9 & 9 & $2,6,9$ & 2,6 & 2,6 & 2,6 & 2 & 2 & 2 & 2 \\
\hline $.5-.5625$ & 2 & $2,8,9$ & 8,9 & 8 & 8,9 & 8,9 & 9 & 2,9 & $2,6,9$ & 2,6 & 2 & 2 & 2 & 2 & 2 & 2 \\
\hline $.4375-.5$ & 2 & $2,8,9$ & 8,9 & 8 & 8,9 & 8,9 & 2,9 & 2,9 & 2 & 2 & 2 & 2 & 2 & 2 & 2 & 2 \\
\hline $.375-.4375$ & 2 & $2,8,9$ & 8,9 & 8 & 8 & $2,8,9$ & 2,9 & 2 & 2 & 2 & 2 & 2 & 2 & 2 & 2 & 2 \\
\hline $.3125-.375$ & 2,8 & $2,8,9$ & 8 & 8 & 8,9 & $2,8,9$ & 2,9 & 2 & 2 & 2 & 2 & 2 & 2 & 2 & 2 & 2 \\
\hline $.25-.3125$ & 2,8 & $2,8,9$ & 8 & 8 & $6,8,9$ & $2,8,9$ & 2 & 2 & 2 & 2 & 2 & 2 & 2 & 2 & 2 & 2 \\
\hline $.1875-.25$ & 2,8 & 8 & 8 & 8 & 2,8 & 2 & 2 & 2 & 2 & 2 & 2 & 2 & 2 & 2 & 2 & 2 \\
\hline $.125-.1875$ & 2,8 & 8 & 8 & 2,8 & 2,8 & 2 & 2 & 2 & 2 & 2 & 2 & 2 & 2 & 2 & 2 & 2 \\
\hline $.0625-.125$ & $1,2,7,8,9$ & 7,8 & 8 & 2,8 & 2,8 & 2 & 2 & 2 & 2 & 2 & 2 & 2 & 2 & 2 & 2 & 2 \\
\hline $0-.0625$ & $1,7,8$ & 7,8 & 7,8 & 184 & 2,8 & 2 & 2 & 2 & 2 & 2 & 2 & 2 & 2 & 2 & 2 & 2 \\
\hline & \begin{tabular}{|c|}
$0-$ \\
0.625
\end{tabular} & \begin{tabular}{|c}
$0.625-$ \\
1.25
\end{tabular} & $\begin{array}{l}1.25- \\
1.875\end{array}$ & $\begin{array}{r}1.875- \\
2.5\end{array}$ & $\begin{array}{l}2.5- \\
3.125\end{array}$ & $\begin{array}{r}3.125- \\
3.75\end{array}$ & $\begin{array}{l}3.75- \\
4.375\end{array}$ & $\begin{array}{c}4.375- \\
5\end{array}$ & $\begin{array}{c}5- \\
5.625\end{array}$ & $\begin{array}{r}5.625- \\
6.25\end{array}$ & $\begin{array}{l}6.25- \\
6.875\end{array}$ & $\begin{array}{r}6.875- \\
7.5\end{array}$ & $\begin{array}{c}7.5- \\
8.125\end{array}$ & $\begin{array}{r}8.125- \\
8.75\end{array}$ & $\begin{array}{l}8.75- \\
9.375\end{array}$ & $\begin{array}{r}9.375 \\
10\end{array}$ \\
\hline
\end{tabular}

\section{Mean Curvature}

Figure 22. Chart showing which tests were used in each input rectangle. In some rectangles there were several subdivisions, and several tests were used. Steps 3, 4 and 5 were never used to reject input rectangles.

Acknowledgements. We are indebted to Frank Morgan for introducing us to this problem, to John Sullivan for generously providing us with computer graphics of torus and double bubbles, and to F. Morgan, M. Hutchings, W. Kahan and W. Rossman for helpful discussions.

University of California, Davis, CA

http://math.ucdavis.edu/hass

E-mail address: hass@math.ucdavis.edu

Dept. of Computer Science, University of California, Santa Cruz, Ca

http://bbs.cruzio.com/schlafly

E-mail address: real@ieee.org

\section{References}

[1] M. Alfaro, J. Brock, J. Foisy, N. Hodges, and J. Zimba, The standard double soap bubble in $R^{2}$ uniquely minimizes perimeter, Pacific J. Math. 159 (1993), 47-59.

[2] G. Alefeld and J. Herzberger, Introduction to Interval Computations, Academic Press, Inc., New York, 1983.

[3] F. J. Almgren, Existence and regularity almost everywhere of solutions to elliptic variational problems with constraints, Memoirs Amer. Math. Soc. 4 (1976), 165-199. 
[4] F. J. Almgren and J. Taylor, The geometry of soap films and soap bubbles, Sci. Amer. 235 (1976), 82-93.

[5] ANSI/IEEE Standard 754-1985 for Binary Floating-Point Arithmetic, The Institute of Electrical and Electronic Engineers, New York, 1985.

[6] K. Appel and W. Haken, The solution of the four-color-map problem, Sci. Amer. 237 (1977), 108-121.

[7] M. Athanassenas, A free boundary problem for capillary surfaces, Manuscripta Math. 76 (1992), 5-19.

[8] J. L. BARBosa and M. Do CARMo, Stability of hypersurfaces with constant mean curvature, Math. Z. 185 (1984), 339-353.

[9] M. Berger and B. Gostiaux, Differential Geometry, Manifolds Curves and Surfaces, Springer-Verlag Graduate Texts 115, Springer-Verlag, New York (1988).

[10] C. V. Boys, Soap Bubbles, Dover Publ. Inc., New York, 1959 (first edition 1911).

[11] Carthage, Encyclopedia Britannica, 19th Edition, 1929.

[12] R. DE la Llave, Computer assisted proofs of stability of matter, in Computer Aided Proofs in Analysis (Cincinnati, OH, 1989), 116-126, IMA Vol. Math. Appl. 28, Springer-Verlag, New York, 1991.

[13] C. Delaunay, Sur la surface de revolution dont la courbure moyenne est constante, $J$. Math. Pure et Appl. 16 (1841), 309-321.

[14] J. Eells, The surfaces of Delaunay, Math. Intelligencer 9 (1987), 53-57.

[15] C. Fefferman, The $N$-body problem in quantum mechanics, Comm. Pure Applied Math. 39 (1986), S67-S109; Addendum, ibid., 40 (1987), 523.

[16] J. Foisy, Soap bubble clusters in $R^{2}$ and $\mathbb{R}^{3}$, undergraduate thesis, Williams College, 1991.

[17] D. Gabai, R. Meyerhoff, and N. Thurston, Homotopy hyperbolic 3-manifolds are hyperbolic, Ann. of Math., to appear.

[18] R. Gulliver, Regularity of minimizing surfaces of prescribed mean curvature, Ann. of Math. 97 (1973), 275-305.

[19] T. Hales, The Kepler conjecture, E-print math.MG/9811078.

[20] J. Hass, M. Hutchings, and R. Schlafly, The double bubble conjecture, ERA Amer. Math. Soc. 1 (1995), 98-102.

[21] J. Hass and R. Schlafly, C++ source code for calculations in the paper Double bubbles minimize; http://www.aom.princeton.edu/00/march/doubbub.cpp .

[22] M. Hutchings, The structure of area-minimizing double bubbles, J. Geom. Anal. 7 (1997), 285-304.

[23] K. Kenmotsu, Surfaces of revolution with prescribed mean curvature, Tôhoku Math. J. 32 (1980), 147-153.

[24] Lord Kelvin and W. Thomson, On the division of space with minimum partitional area, Phil. Mag. 24 (1887), 503.

[25] N. Korevaar, R. Kusner, and B. Solomon, The structure of complete embedded surfaces with constant mean curvature, J. Differential Geom. 30 (1989), 465-503.

[26] G. Lawlor and F. Morgan, Paired calibrations applied to soap films, immiscible fluids, and surfaces or networks minimizing other norms, Pacific J. Math. 166 (1994), 55-83.

[27] O. Lanford, Proof of the Feigenbaum conjectures, Bull. Amer. Math. Soc. 6 (1982), 427-434.

[28] H. P. McKean, M. Schreiber, and G. H. Weiss, Isoperimetric problem with application to the figure of cells, J. Math. Physics 6 (1994), 479-484.

[29] R. E. Moore, Methods and applications of interval analysis, SIAM Studies in Applied Math. 2, SIAM, Philadelphia, PA, 1979.

[30] J. D. Moore and R. Schlafly, On equivariant isometric embeddings, Math Z 173 (1980), 119-129.

[31] F. Morgan, Soap bubbles in $R^{2}$ and in surfaces, Pacific J. Math. 165 (1994), 347-361. 
[32] F. Morgan, Clusters minimizing area plus length of singular curves, Math. Ann. 299 (1994), 697-714.

[33] R. Osserman, The isoperimetric inequality, Bull. Amer. Math. Soc. 84 (1978), 1182-1238.

[34] R. S. Mackay and I. C. Percival, Converse KAM: theory and practice, Comm. Math. Phys. 98 (1985), 469-512.

[35] J. Plateau, Statique Experimentale et Theorique des Liquides Soumis auz Seules Forces Molecularies, Gathier-Villars, Paris, 1873.

[36] H. SAGAN, Introduction to the Calculus of Variations, Dover Publications Inc., New York, 1992.

[37] N. Robertson, D. Sanders, P. Seymour, and R. Thomas, The four-colour theorem, J. Combin. Theory 70 (1997), 2-44.

[38] M. SpIvak, A Comprehensive Introduction to Differential Geometry, Volumes III and IV, Second Edition, Publish or Perish Inc., Wilmington, Del., 1979.

[39] J. TAYLOR, The structure of singularities in soap-bubble-like and soap-film-like minimal surfaces, Ann. of Math. 103 (1976), 489-539.

[40] D. Thompson, On Growth and Form, Cambridge Univ. Press, Cambridge, 1959 (first edition 1917).

[41] T. I. Vogel, Stability of a liquid drop trapped between two parallel planes, SIAM J. Appl. Math. 47 (1987), 516-525.

(Received December 29, 1995)

(Revised August 2, 1999) 


$$
8
$$

